\title{
NMSSM with generalized deflected mirage mediation
}

\author{
Xiao Kang Du ${ }^{1}$, Guo-Li Liu ${ }^{1}$, Fei Wang ${ }^{1, a}$, Wenyu Wang ${ }^{2}$, Jin Min Yang ${ }^{3}$, Yang Zhang ${ }^{4}$ \\ ${ }^{1}$ School of Physics, Zhengzhou University, ZhengZhou 450000, People's Republic of China \\ ${ }^{2}$ School of Physics, Beijing University of Technology, Beijing, People's Republic of China \\ ${ }^{3}$ CAS Key Laboratory of Theoretical Physics, Institute of Theoretical Physics, Chinese Academy of Sciences, Beijing 100190, \\ People's Republic of China \\ ${ }^{4}$ ARC Centre of Excellence for Particle Physics at the Tera-scale, School of Physics and Astronomy, Monash University, Melbourne, VIC 3800, \\ Australia
}

Received: 18 December 2018 / Accepted: 27 April 2019 / Published online: 9 May 2019

(C) The Author(s) 2019

\begin{abstract}
We propose to generate a realistic soft SUSY breaking spectrum for Next-to-Minimal Supersymmetric Standard Model (NMSSM) with a generalized deflected mirage mediation scenario, in which additional Yukawa and gauge mediation contributions are included to deflect the renormalization group equation trajectory. Based on the Wilsonian effective action obtained by integrating out the messengers, the NMSSM soft SUSY breaking spectrum can be given analytically at the messenger scale. We find that additional contributions to $m_{S}^{2}$ can possibly ameliorate the stringent constraints from the electroweak symmetry breaking and $125 \mathrm{GeV}$ Higgs mass. Constraints from dark matter and fine-tuning are also discussed. The Barbieri-Giudice fine-tuning measure and electroweak fine-tuning measure in our scenario can be as low as $\mathcal{O}(1)$, which possibly indicates that our scenario is natural.
\end{abstract}

\section{Contents}

1 Introduction .................. 1

2 Brief review of the mirage mediation mechanism . . 2

3 NMSSM with deflected mirage mediation . . . . . . 3

3.1 The modular weight choices for NMSSM superfields . . . . . . . . . . . . . 4

3.2 Analytical expressions for soft SUSY breaking parameters . . . . . . . . . . 4

4 Numerical results . . . . . . . . . . . . . . . 8

5 Conclusions . . . . . . . . . . . 15

Appendix A: The mirage scale in deflected mirage mediation mechanism . . . . . . . . . . . . . 16

References . . . . . . . . . . . . . . . . 16

a e-mail: feiwang@zzu.edu.cn

\section{Introduction}

Low energy supersymmetry (SUSY), which can solve elegantly the gauge hierarchy problem of the Standard Model (SM) and provide a viable dark matter (DM) candidate, has been regarded by many physicists as one of the most appealing candidates for the TeV-scale new physics. However, the reported data of the Large Hadron Collider (LHC) agree quite well with the SM predictions and no significant deviations have been observed in the electroweak precision measurements and the flavor physics. Besides, the lack of SUSY signals at the LHC [1-3] and the difficulty to accommodate the discovered $125 \mathrm{GeV}$ Higgs $[4,5]$ seem to indicate that the low energy SUSY spectrum should display an intricated structure. As the low energy soft SUSY breaking spectrum is determined by the SUSY breaking mechanism, it is interesting to survey the phenomenology related to the SUSY breaking and mediation mechanism from a top-down approach.

SUSY breaking mechanism from flux compactification of type IIB string theory can lead to interesting consequences. In the generalized Kahler-modulus dominated scenarios, the dilaton and complex structure moduli fields can be stabilized by the background NS and RR 3-form fluxes. Such superheavy modes will be integrated out and eliminated from the low energy effective theory. The remaining Kahler moduli fields can be stabilized by non-perturbative effects, such as the gaugino condensation. To generate SUSY breaking in the observable sector and tune the cosmological constant to a tiny positive value, Kachru et al. propose to add an anti-D3 brane at the tip of the Klebanov-Strassler throat to explicitly break SUSY and lift the AdS universe to obtain a dS one [6]. Consequently, the F-component of the light Kahler moduli field could mediate the SUSY breaking effects to the visible sector and result in a mixed modulus-anomaly mediation SUSY breaking scenario $[7,8]$. It is interesting to note that the 
involved modulus mediated SUSY breaking contributions can be comparable to that of the anomaly mediation $[9,10]$. With certain assumptions on the Yukawa couplings and the modular weights, the change of the SUSY breaking contributions (from the inputs) by the renormalization group equation (RGE) evolution and the anomaly mediation contributions could cancel each other at a 'mirage' scale, leading to an apparent pure modulus mediation scenario at such a mirage scale [11]. Such a mixed modulus-anomaly mediation SUSY breaking mechanism is dubbed as 'mirage mediation'.

Anomaly mediation contribution is a crucial ingredient of such a mixed modulus-anomaly mediation mechanism. It is well known that purely anomaly mediation mechanism is bothered by the tachyonic slepton problem [12-18]. One of its non-trivial extensions with a messenger sector, namely the deflected anomaly mediation SUSY breaking (AMSB) scenario, can elegantly solve such a tachyonic slepton problem through the deflection of the RGE trajectory [19-25] by additional gauge [26-32] or Yukawa mediation contributions. Such a messenger sector can also be present in the mirage mediation scenarios and play an important role in generating a preferable low energy SUSY spectrum. In fact, the 'mirage' unification of gaugino masses at $\mathrm{TeV}$ scale can be possible in deflected mirage mediation scenarios, even with the simplest minimal KKLT set-up [33-35]. In the mixed modulus-anomaly mediation mechanism, a straightforward extension of $\mu$ sector to predict a small $B \mu$ term needs some fine tuning [11]. Such a $\mu-B \mu$ problem can be solved naturally in the framework of Next-to-Minimal Supersymmetric Standard Model (NMSSM). The realization of NMSSM in $\mathrm{TeV}$ mirage mediation scenarios had already been discussed in the literature [36-39]. However, it was found that only a small portion of the parameter space can be consistent with the electroweak symmetry breaking (EWSB) conditions and at the same time accommodate the $125 \mathrm{GeV}$ Higgs [36,37]. So it is rather interesting to seek new ways to generate a realistic NMSSM spectrum.

Additional gauge or Yukawa mediation contributions in the mirage mediation [33-35] can deflect the RGE trajectory and change the low energy soft SUSY predictions. Analytical expressions for the soft SUSY breaking parameters at the messenger scale are given in the Wilsonian effective action approach [40] by one of the authors. Based on the general discussions in [40], we propose to generate the NMSSM spectrum by a generalized deflected mirage mediation mechanism [41] with additional gauge and Yukawa mediation contributions. We find that the inclusion of the messenger sector and non-trivial interactions can possibly alleviate the stringent constraints from the $125 \mathrm{GeV}$ Higgs and the EWSB conditions.

This paper is organized as follows. We briefly review the mirage mediation mechanism in Sect. 2. We state our model and present the analytical expressions for the soft SUSY parameters in Sect. 3. The numerical results are discussed in Sect. 4. Section 5 contains our conclusions.

\section{Brief review of the mirage mediation mechanism}

In Type IIB string theory compactified on a Calabi-Yau orientifold, the presence of background fluxes can fix the dilaton and the complex structure moduli, leaving only the Kahler moduli in the four-dimensional Wilsonian effective supergravity action (defined at the boundary scale $\Lambda$ ) after integrating out the superheavy complex structure moduli and dilaton. The low energy effective Lagrangian in terms of compensator field and a single Kahler modulus that parameterizes the overall size of the compact space [11] is given as

$$
\begin{aligned}
e^{-1} \mathcal{L}= & \int d^{4} \theta\left[\phi^{\dagger} \phi\left(-3 e^{-K / 3}\right)-\left(\phi^{\dagger} \phi\right)^{2} \bar{\theta}^{2} \theta^{2} \mathcal{P}_{\text {lift }}\right] \\
& +\int d^{2} \theta \phi^{3} W+\int d^{2} \theta \frac{f_{i}}{4} W_{i}^{a} W_{i}^{a},
\end{aligned}
$$

with a holomorphic gauge kinetic term

$f_{i}=\frac{1}{g_{i}^{2}}+i \frac{\theta}{8 \pi}$.

The Kahler potential involves the 'no-scale' kinetic form for the Kahler modulus while the superpotential involves the KKLT setup

$W=\left(\omega_{0}-A e^{-a T}\right)+W_{0}$,

where the first term is generated from the fluxes and the second term from non-perturbative effects, such as gaugino condensation from the non-abelian gauge sector or D3-brane instantons. $W_{0}$ denotes the superpotential terms involving the MSSM (NMSSM) sector as well as a possible messenger sector. The modulus $T$, which is not fixed by the background flux, can be stabilized by such a KKLT-type superpotential with

$a \Re\langle T\rangle \approx \ln \left(\frac{A}{\omega_{0}}\right) \approx \ln \left(\frac{M_{P l}}{m_{3 / 2}}\right) \approx 4 \pi^{2}$,

up to $\mathcal{O}\left(\ln \left[M_{P l} / m_{3 / 2}\right]^{-1}\right)$. Note that in the KKLT setup, the flux-induced SUSY breaking is dynamically canceled by the non-perturbative SUSY breaking that stabilizes the Kahler modulus $T$, leading to a SUSY-preserving AdS solution. In order to obtain a vacuum with a positive cosmological constant and break SUSY, KKLT proposed to add an $\bar{D} 3$ brane to provide an uplifting operator given by

$\mathcal{P}_{\text {lift }}=D\left(T+T^{\dagger}\right)^{n_{P}}$,

with a positive constant $D \sim \mathcal{O}\left(m_{3 / 2}^{2} M_{p l}^{2}\right)$. The uplifting operator, which represents the low energy consequence of the sequestered SUSY-breaking brane, is independent of visible matter fields and $T$ (with $n_{P}=0$ ) in the minimal KKLT 
set-up. Explicit SUSY breaking via anti-D3 branes can be replaced by typical D-term or F-term uplifting mechanisms [42-44].

With the uplifting low energy effective potential, we have the leading order F-terms of compensator field $F_{\phi}$ and Kahler modulus $F_{T}$ [11]

$$
\begin{aligned}
& F_{\phi} \approx m_{3 / 2} \approx \frac{\omega_{0}}{M_{P l}^{2}\left(T+T^{*}\right)^{3 / 2}}, \\
& M_{0} \equiv \frac{F_{T}}{T+T^{*}} \approx \frac{2}{a\left(T+T^{*}\right)} m_{3 / 2} \approx \frac{m_{3 / 2}}{\ln \left(\frac{M_{P l}}{m_{3 / 2}}\right)} .
\end{aligned}
$$

The non-zero F-term VEV of the heavy moduli $H$ are given approximately by $F_{H} \sim m_{3 / 2}^{2} / m_{U} \ll m_{3 / 2}$. Therefore, it gives negligible contributions to SUSY breaking [45].

In the mirage mediation, we have $m_{3 / 2} \approx\left(4 \pi^{2}\right) M_{0}$ numerically, which means that the loop induced anomaly mediation contributions are comparable to the modulus mediation contributions. The importance of the anomaly mediation contributions relative to the modulus mediation contributions can be parameterized by

$\alpha^{\prime} \equiv \frac{m_{3 / 2}}{M_{0} \ln \left(M_{P l} / m_{3 / 2}\right)}$

So $\alpha^{\prime} \ll 1$ corresponds to the limit of pure modulus mediation, while $\alpha^{\prime} \gg 1$ corresponds to the pure anomaly mediation. Although the minimal KKLT predicts $\alpha^{\prime} \approx 1$, other values of $\alpha^{\prime} \sim \mathcal{O}(1)$ can be obtained by assuming proper uplifting operator and proper forms for the potential of Kahler modulus [11]. So we leave the value $\alpha^{\prime}$ as a free parameter in the following discussions.

\section{NMSSM with deflected mirage mediation}

The general form of the low energy effective action Eq. (2.1) can be amended to include new ingredients, for example, the NMSSM sector and a messenger sector. The Kahler potential will include not only the 'no-scale' kinetic form for the Kahler modulus but also additional kinetic terms for messenger fields

$$
\begin{aligned}
K= & -3 \ln \left(T+T^{\dagger}\right)+Z_{X}\left(T^{\dagger}, T\right) X^{\dagger} X+Z_{\Phi}\left(T^{\dagger}, T\right) \Phi^{\dagger} \Phi \\
& +\sum_{i} Z_{P_{i}, \bar{P}_{i}}\left(T^{\dagger}, T\right)\left[P_{i}^{\dagger} P_{i}+\bar{P}_{i}^{\dagger} \bar{P}_{i}\right],
\end{aligned}
$$

with $\bar{P}_{i}, P_{i}$ the messenger superfields and $\Phi$ the NMSSM superfield. The Kahler metric for matter fields and messengers as well as holomorhic gauge kinetic functions are assumed to depend non-trivially on the Kahler modulus $T$
$Z_{X}\left(T^{\dagger}, T\right)=\frac{1}{\left(T^{\dagger}+T\right)^{n_{X}}}, \quad Z_{\Phi}\left(T^{\dagger}, T\right)=\frac{1}{\left(T^{\dagger}+T\right)^{n_{\Phi}}}$,

$f_{i}(T)=T^{l_{i}}, \quad Z_{P_{i}, \bar{P}_{i}}\left(T^{\dagger}, T\right)=\frac{1}{\left(T^{\dagger}+T\right)^{n_{P}}}$.

The values of $n_{X}, n_{\Phi}, n_{P}, l_{i}$ depend on the location of the fields on the D3/D7 branes. Besides, universal $l_{i}=1$ are adopted in our scenario so that the gauge fields reside on the D7 brane and the gauge coupling unification can be preserved.

The $W_{0}$ term within the superpotential Eq. (2.3) is given as

$W_{0}=W_{\overline{N M S S M}}+W_{M}$,

with

$W_{\overline{N M S S M}}=\lambda S H_{u} H_{d}+\frac{1}{3} \kappa S^{3}+W_{M S S M}$,

the $\left(Z_{3}\right.$ symmetric) NMSSM sector and

$$
\begin{aligned}
W_{M}= & \sum_{m}\left[\lambda_{X}^{T} X \tilde{X}_{m} X_{m}+\lambda_{X}^{D} X \tilde{Y}_{m} Y_{m}\right] \\
& +\lambda_{P}^{T} S \tilde{X}_{1} X_{2}+\lambda_{P}^{D} S \tilde{Y}_{1} Y_{2}+W(X),
\end{aligned}
$$

the messenger sector. The ' $2 m$ ' family of messengers can be fitted into $\mathbf{5}, \overline{\mathbf{5}}$ representation of $S U(5)$ gauge group

$P_{m}(\mathbf{5})=X_{m}(3,1)_{-1 / 3} \oplus Y_{m}(1,2)_{1 / 2}$,

$\tilde{P}_{m}(\overline{\mathbf{5}})=\bar{X}_{m}(\overline{3}, 1)_{1 / 3} \oplus \bar{Y}_{m}(1, \overline{2})_{-1 / 2}$.

The VEV of the pseudo-modulus superfield $X$, which can be determined by $W(X)$ and other SUSY breaking effects, can set the messenger thresholds through the $X P_{m} \tilde{P}_{m}$ type couplings. The deflection parameter ' $d$ ', which characterizes the relative size of contributions between the gauge (Yukawa) mediation and anomaly mediation, can be readily obtained

$d F_{\phi} \equiv \frac{F_{X}}{X}-F_{\phi}$

Similar to the deflected AMSB, a positive deflection parameter in the deflected mirage mediation, which can be generated by a carefully chosen superpotential etc. [21-25], may be preferable because less messenger species are needed so that the problem of strong gauge couplings below the GUT scale can be evaded. The purpose to introduce even number of messenger species is to forbid possible kinetic mixing between $X$ and $S$, otherwise the tadpole term for $S$ would destabilize the weak scale [46]. The discrete $Z_{3}$ breaking by EWSB may generate domain walls in the early universe which may lead to an unacceptably large anisotropy of CMB. To avoid such a problem, the $Z_{3}$ symmetry is assumed to be broken by some higher dimensional operators.

There are two approaches to obtain the low energy SUSY spectrum in the deflected mirage mediation: 
- In the first approach, the deflected mirage mediation soft SUSY spectrum is given by the expressions in [11] at the boundary scale. Such a spectrum will receive additional contributions towards its RGE running to low energy scale, especially the threshold corrections related to the appearance of messengers [47].

- In the second approach which we will adopt, the soft SUSY spectrum at low energy scale is derived directly from the (low energy) effective action. The SUGRA description in Eq. (2.1) can be seen as the Wilsonian effective action after integrating out the superheavy complex structure moduli and dilaton field. After the pseudomodulus $X$ acquires a VEV and determines the messenger threshold, the messengers can be integrated out to obtain a low energy effective action below the messenger threshold scale. So we anticipate that the Kahler metric $Z_{\Phi}$ and gauge kinetic $f_{i}$ should depend non-trivially on the messenger threshold $M_{m e s s}^{2} / \phi^{\dagger} \phi$ and $M_{m e s s} / \phi$, respectively. The resulting soft SUSY spectrum below the messenger threshold can be derived from the wavefunction renormalization approach [48-54]. The analytical expressions for the soft SUSY breaking parameters in the most general form of deflected mirage mediation scenarios are given in [40] by one of the authors.

\subsection{The modular weight choices for NMSSM superfields}

We need to specify the modular weights $n_{i} \equiv 1-m_{i}$ for NMSSM superfields before we can carry out numerical analysis. In the NMSSM, successful EWSB and the solution to the $\mu$-problem in general require a large VEV for the singlet $S$. So it is preferable to introduce a negative $m_{S}^{2}$ and/or large trilinear terms $A_{\lambda}, A_{\kappa}$ for the singlet superpotential interactions. As a negative $m_{S}^{2}$ prefers vanishing modulus contributions, we set the modular weight $m_{S}=0$. The choice of modulus weight for $H_{u}, H_{d}$ can be understood from the EWSB conditions in NMSSM. From

$\frac{M_{Z}^{2}}{2}=\frac{m_{H_{d}}^{2}-m_{H_{u}}^{2} \tan \beta^{2}}{\tan ^{2} \beta-1}-\mu^{2}$,

we can see that $m_{H_{u}}^{2}$ should be light to avoid a too large finetuning. On the other hand, $m_{H_{u}}^{2} \ll m_{H_{d}}^{2}$ for $\tan \beta \gg 1$. So we can set $m\left(H_{d}\right)=1$ or $1 / 2$ and $m_{H_{u}}=0$.

The electroweak (EW) naturalness prefers relatively light stops. In the MSSM, light stops below $1 \mathrm{TeV}$ are disfavored because it is difficult to accommodate the observed $125 \mathrm{GeV}$ Higgs. However, large loop corrections involving stops are not necessarily required to interpret the $125 \mathrm{GeV}$ Higgs in NMSSM. So light stops, which is preferable from the criterion of a low EW fine-tuning, are still allowed in NMSSM. Squarks of the first two generations should be heavy to avoid various SUSY CP and flavor constraints. We note that even pure AMSB contributions can guarantee the heaviness of colored SUSY particles. Besides, it is preferable to ameliorate the $g_{\mu}-2$ discrepancy in the framework of SUSY with light sleptons and electroweakinoes. With the modular weight $l_{i}=1$ for gauge fields, a positive deflection parameter ' $d$ ' can possibly guarantee the lightness of the electroweakinos.

The notorious tachyonic slepton problem in AMSB can be solved in our scenario. Positive slepton masses can be obtained by introducing a proper deflection parameter ' $d$ ' or by adding extra modulus mediation contributions. So we chose the following $m_{i}$ in our scenario:

- Modular weights for sleptons are given by $m_{\left(L_{L}\right)^{1,2,3}}=$ $m_{\left(E_{L}^{c}\right)^{1,2,3}}=1 / 2$.

- Modular weights for other matter and messenger fields are given by

$$
\begin{aligned}
& m_{H_{u}}=m_{S}=m_{Q_{L}^{3}}=m_{t_{L}^{c}}=m_{b_{L}^{c}}=0 \\
& m_{a}=\frac{1}{2}, \quad\left(a=Q_{L}^{1,2},\left(U_{L}^{c}\right)^{1,2},\left(D_{L}^{c}\right)^{1,2}\right), \\
& m_{H_{d}}=m_{X}=m_{\tilde{X}}=m_{Y}=m_{\tilde{Y}}=1 .
\end{aligned}
$$

- Double messenger species with $m=1$ are adopted in our subsequent numerical study.

Note that the messenger modular weights also play a role and contribute to $m_{S}^{2}$. The modular weights $n_{i}=0$ correspond to matter fields on D7 branes while $n_{i}=1$ on D3 branes. Modular weights $n_{i}=1 / 2$ corresponds to fields on the intersections of the D3-D7 branes.

\subsection{Analytical expressions for soft SUSY breaking parameters}

Now we use the second Wilsonian approach in our analysis. From the analytical expressions for the generalized mirage mediation [40], the soft SUSY breaking parameters in NMSSM at the messenger scale $M_{\text {mess }}$ after integrating out the messenger fields can be given explicitly. The gaugino masses are given as

$M_{i}=l_{i} M_{0} \frac{g_{i}^{2}(\mu)}{g_{i}^{2}(G U T)}+F_{\phi} \frac{\alpha_{i}}{4 \pi}\left(b_{i}-d \Delta b_{i}\right)$,

with $l_{i}=1$ and

$\left(b_{1}, b_{2}, b_{3}\right)=\left(\frac{33}{5}, 1,-3\right)$,

$\Delta\left(b_{1}, b_{2}, b_{3}\right)=(2,2,2)$.

Within the expression, the relative size between the anomaly and modulus mediation contribution at the messenger scale 
is determined by the free parameter

$\alpha=\frac{F_{\phi}}{\left(16 \pi^{2}\right) M_{0}}$.

We define the modular weight $q_{y_{i j k}}$ as

$$
\begin{aligned}
q_{y_{t}} & =m_{Q_{L, 3}}+m_{H_{u}}+m_{t_{L}^{c}}, \\
q_{y_{b}} & =m_{Q_{L, 3}}+m_{H_{d}}+m_{b_{L}^{c}}, \\
q_{y_{\tau}} & =m_{L_{L, 3}}+m_{H_{d}}+m_{\tau_{L}^{c}}, \\
q_{\lambda} & =m_{S}+m_{H_{d}}+m_{H_{u}}, \\
q_{\kappa} & =3 m_{S} .
\end{aligned}
$$

The trilinear soft terms at the messenger scale are given by

$$
\begin{aligned}
& A_{t}=q_{y_{t}} M_{0}-\frac{M_{0}}{8 \pi^{2}}\left[6 y_{t}^{2} \frac{q_{y_{t}}}{2}+y_{b}^{2} \frac{q_{y_{b}}}{2}+\lambda^{2} \frac{q \lambda}{2}\right. \\
& \left.-\left(\frac{16}{3} g_{3}^{2}+3 g_{2}^{2}+\frac{13}{15} g_{1}^{2}\right)\right] \ln \left(\frac{M_{G U T}}{M_{m e s s}}\right) \\
& +\frac{F_{\phi}}{16 \pi^{2}}\left[6 y_{t}^{2}+y_{b}^{2}+\lambda^{2}-\left(\frac{16}{3} g_{3}^{2}+3 g_{2}^{2}+\frac{13}{15} g_{1}^{2}\right)\right] \text {, } \\
& A_{b}=q_{y_{b}} M_{0}-\frac{M_{0}}{8 \pi^{2}}\left[y_{t}^{2} \frac{q_{y_{t}}}{2}+6 y_{b}^{2} \frac{q_{y_{b}}}{2}+\lambda^{2} \frac{q \lambda}{2}\right. \\
& \left.-\left(\frac{16}{3} g_{3}^{2}+3 g_{2}^{2}+\frac{7}{15} g_{1}^{2}\right)\right] \ln \left(\frac{M_{G U T}}{M_{m e s s}}\right) \\
& +\frac{F_{\phi}}{16 \pi^{2}}\left[y_{t}^{2}+6 y_{b}^{2}+\lambda^{2}-\left(\frac{16}{3} g_{3}^{2}+3 g_{2}^{2}+\frac{7}{15} g_{1}^{2}\right)\right] \text {, } \\
& A_{\tau}=q_{y_{\tau}} M_{0}-\frac{M_{0}}{8 \pi^{2}}\left[3 y_{b}^{2} \frac{q_{y_{b}}}{2}+\lambda^{2} \frac{q \lambda}{2}\right. \\
& \left.-\left(3 g_{2}^{2}+\frac{9}{5} g_{1}^{2}\right)\right] \ln \left(\frac{M_{G U T}}{M_{\text {mess }}}\right) \\
& +\frac{F_{\phi}}{16 \pi^{2}}\left[3 y_{b}^{2}+\lambda^{2}-\left(3 g_{2}^{2}+\frac{9}{5} g_{1}^{2}\right)\right] \text {, } \\
& A_{\lambda}=q_{\lambda} M_{0}-\frac{M_{0}}{8 \pi^{2}}\left[3 y_{t}^{2} \frac{q_{y_{t}}}{2}+3 y_{b}^{2} \frac{q_{y_{b}}}{2}+4 \lambda^{2} \frac{q \lambda}{2}\right. \\
& \left.+2 \kappa^{2} \frac{q_{\kappa}}{2}-\left(3 g_{2}^{2}+\frac{3}{5} g_{1}^{2}\right)\right] \ln \left(\frac{M_{G U T}}{M_{m e s s}}\right) \\
& +\frac{F_{\phi}}{16 \pi^{2}}\left[4 \lambda^{2}+2 \kappa^{2}+3 y_{t}^{2}+3 y_{b}^{2}-\left(3 g_{2}^{2}+\frac{3}{5} g_{1}^{2}\right)\right] \\
& +\Delta A_{\lambda} \\
& A_{\kappa}=q_{\kappa} M_{0}-\frac{M_{0}}{8 \pi^{2}}\left[6 \lambda^{2} \frac{q \lambda}{2}+6 \kappa^{2} \frac{q_{\kappa}}{2}\right] \ln \left(\frac{M_{G U T}}{M_{m e s s}}\right) \\
& +\frac{F_{\phi}}{16 \pi^{2}}\left[6 \lambda^{2}+6 \kappa^{2}\right]+\Delta A_{\kappa},
\end{aligned}
$$

with new contributions due to non-vanishing $\Delta G_{S}$

$$
\begin{aligned}
\Delta A_{\lambda} & =-d \frac{F_{\phi}}{16 \pi^{2}}\left[3\left(\lambda_{P}^{T}\right)^{2}+2\left(\lambda_{P}^{D}\right)^{2}\right], \\
\Delta A_{\kappa} & =-3 d \frac{F_{\phi}}{16 \pi^{2}}\left[3\left(\lambda_{P}^{T}\right)^{2}+2\left(\lambda_{P}^{D}\right)^{2}\right] .
\end{aligned}
$$

The soft SUSY breaking parameters for the scalars can be parameterized by

$m_{\text {soft }}^{2}=\delta_{m}+\delta_{d}+\delta_{I}$,

with each part given as follows

- The pure modulus contribution part

$$
\begin{aligned}
\delta_{\tilde{Q}_{L ; 3}}^{m}= & m_{Q_{L ; 3}} M_{0}^{2} \\
& -\frac{M_{0}^{2}}{8 \pi^{2}} \ln \left(\frac{M_{G U T}}{M_{m e s s}}\right)\left\{\left(q_{y_{t}}^{2}+q_{y_{t}}\right) y_{t}^{2}+\left(q_{y_{b}}^{2}\right.\right. \\
& \left.+q_{y_{b}}\right) y_{b}^{2}-\frac{16}{3} g_{3}^{2}-3 g_{2}^{2}-\frac{1}{15} g_{1}^{2} \\
& +\frac{1}{8 \pi^{2}}\left[-y_{t}^{2} q_{y_{t}} K_{y_{t}}-y_{b}^{2} q_{y_{b}} K_{y_{b}}\right] \\
& +\frac{1}{8 \pi^{2}}\left[\frac{8}{3} b_{3}^{\prime} g_{3}^{2}+\frac{3}{2} b_{2}^{\prime} g_{2}^{2}+\frac{1}{30} b_{1}^{\prime} g_{1}^{2}\right] \\
& \left.\times \ln \left(\frac{G U T}{M_{m e s s}}\right)\right\}, \\
\delta_{\tilde{U}_{L ; 3}^{c}}^{m}= & m_{U_{L ; 3}^{c}} M_{0}^{2}-\frac{M_{0}^{2}}{8 \pi^{2}} \ln \left(\frac{M_{G U T}}{M_{m e s s}}\right)\left\{2\left(q_{y_{t}}^{2}+q_{y_{t}}\right) y_{t}^{2}\right. \\
& -\frac{16}{3} g_{3}^{2}-\frac{16}{15} g_{1}^{2} \\
& +\frac{1}{8 \pi^{2}}\left[-2 y_{t}^{2} q_{y_{t}} K_{y_{t}}\right] \\
& \left.+\frac{1}{8 \pi^{2}}\left[\frac{8}{3} b_{3}^{\prime} g_{3}^{2}+\frac{8}{15} b_{1}^{\prime} g_{1}^{2}\right] \ln \left(\frac{G U T}{M_{m e s s}}\right)\right\},
\end{aligned}
$$

$$
\begin{aligned}
\delta_{\tilde{D}_{L ; 3}^{c}}^{m}= & m_{D_{L ; 3}^{c}} M_{0}^{2}-\frac{M_{0}^{2}}{8 \pi^{2}} \ln \left(\frac{M_{G U T}}{M_{\text {mess }}}\right)\left\{2\left(q_{y_{b}}^{2}+q_{y_{b}}\right) y_{b}^{2}\right. \\
& -\frac{16}{3} g_{3}^{2}-\frac{4}{15} g_{1}^{2} \\
& +\frac{1}{8 \pi^{2}}\left[-2 y_{b}^{2} q_{y_{b}} K_{y_{b}}\right] \\
& \left.+\frac{1}{8 \pi^{2}}\left[\frac{8}{3} b_{3}^{\prime} g_{3}^{2}+\frac{2}{15} b_{1}^{\prime} g_{1}^{2}\right] \ln \left(\frac{G U T}{M_{\text {mess }}}\right)\right\},
\end{aligned}
$$$$
\delta_{\tilde{L}_{L ; a}}^{m}=m_{L_{L ; a}} M_{0}^{2}
$$$$
-\frac{M_{0}^{2}}{8 \pi^{2}} \ln \left(\frac{M_{G U T}}{M_{\text {mess }}}\right)\left\{-3 g_{2}^{2}-\frac{3}{5} g_{1}^{2}\right.
$$$$
\left.+\frac{1}{8 \pi^{2}}\left[\frac{3}{2} b_{2}^{\prime} g_{2}^{2}+\frac{3}{10} b_{1}^{\prime} g_{1}^{2}\right] \ln \left(\frac{G U T}{M_{\text {mess }}}\right)\right\} \text {, }
$$

$$
\begin{aligned}
\delta_{\tilde{E}_{L ; a}^{c}}^{m}= & m_{E_{L ; a}^{c}} M_{0}^{2} \\
& -\frac{M_{0}^{2}}{8 \pi^{2}} \ln \left(\frac{M_{G U T}}{M_{\text {mess }}}\right)\left\{-\frac{12}{5} g_{1}^{2}\right. \\
& \left.+\frac{1}{8 \pi^{2}}\left(\frac{6}{5} b_{1}^{\prime} g_{1}^{2}\right) \ln \left(\frac{G U T}{M_{\text {mess }}}\right)\right\},
\end{aligned}
$$




$$
\begin{aligned}
\delta_{\tilde{H}_{u}}^{m}= & m_{H_{u}} M_{0}^{2} \\
& -\frac{M_{0}^{2}}{8 \pi^{2}} \ln \left(\frac{M_{G U T}}{M_{\text {mess }}}\right)\left\{3\left(q_{y_{t}}^{2}+q_{y_{t}}\right) y_{t}^{2}\right. \\
& +\left(q_{\lambda}^{2}+q_{\lambda}\right) \lambda^{2}-3 g_{2}^{2}-\frac{3}{5} g_{1}^{2} \\
& +\frac{1}{8 \pi^{2}}\left[-3 y_{t}^{2} q_{y_{t}} K_{y_{t}}-\lambda^{2} q_{\lambda} K_{\lambda}\right] \\
& \left.+\frac{1}{8 \pi^{2}}\left[\frac{3}{2} b_{2}^{\prime} g_{2}^{2}+\frac{3}{10} b_{1}^{\prime} g_{1}^{2}\right] \ln \left(\frac{G U T}{M_{\text {mess }}}\right)\right\}, \\
\delta_{\tilde{H}_{d}}^{m}= & m_{H_{d}} M_{0}^{2}-\frac{M_{0}^{2}}{8 \pi^{2}} \ln \left(\frac{M_{G U T}}{M_{m e s s}}\right)\left\{3\left(q_{y_{b}}^{2}+q_{y_{b}}\right) y_{b}^{2}\right. \\
& +\left(q_{\lambda}^{2}+q_{\lambda}\right) \lambda^{2}-3 g_{2}^{2}-\frac{3}{5} g_{1}^{2} \\
& +\frac{1}{8 \pi^{2}}\left[-3 y_{b}^{2} q_{y_{b}} K_{y_{b}}-\lambda^{2} q_{\lambda} K_{\lambda}\right] \\
& \left.+\frac{1}{8 \pi^{2}}\left[\frac{3}{2} b_{2}^{\prime} g_{2}^{2}+\frac{3}{10} b_{1}^{\prime} g_{1}^{2}\right] \ln \left(\frac{G U T}{M_{\text {mess }}}\right)\right\}, \\
\delta_{S}^{m}= & m_{S} M_{0}^{2}-\frac{M_{0}^{2}}{8 \pi^{2}} \ln \left(\frac{M_{G U T}}{M_{\text {mess }}}\right)\left\{2\left(q_{\lambda}^{2}+q_{\lambda}\right) \lambda^{2}\right. \\
& +2\left(q_{\kappa}^{2}+q_{\kappa}\right) \kappa^{2} \\
& \left.+\frac{1}{8 \pi^{2}}\left[-2 \lambda^{2} q_{\lambda} K_{\lambda}-2 \kappa^{2} q_{\kappa} K_{\kappa}\right]\right\},
\end{aligned}
$$

with $b_{i}^{\prime}=b_{i}+\Delta b_{i}$ being the beta function upon the messenger thresholds and

$$
\begin{aligned}
K_{y_{t}}= & {\left[6 y_{t}^{2} q_{y_{t}}+y_{b}^{2} q_{y_{b}}+\lambda^{2} q_{\lambda}\right.} \\
& \left.-\left(\frac{16}{3} g_{3}^{2}+3 g_{2}^{2}+\frac{13}{15} g_{1}^{2}\right)\right] \ln \left(\frac{G U T}{M_{\text {mess }}}\right), \\
K_{y_{b}}= & {\left[y_{t}^{2} q_{y_{t}}+6 y_{b}^{2} q_{y_{b}}+\lambda^{2} q_{\lambda}\right.} \\
& \left.-\left(\frac{16}{3} g_{3}^{2}+3 g_{2}^{2}+\frac{7}{15} g_{1}^{2}\right)\right] \ln \left(\frac{G U T}{M_{\text {mess }}}\right), \\
K_{\lambda}= & {\left[3 y_{t}^{2} q_{y_{t}}+3 y_{b}^{2} q_{y_{b}}+4 \lambda^{2} q_{\lambda}+2 \kappa^{2} q_{\kappa}\right.} \\
& \left.-\left(3 g_{2}^{2}+\frac{3}{5} g_{1}^{2}\right)\right] \ln \left(\frac{G U T}{M_{\text {mess }}}\right), \\
K_{\kappa}= & {\left[6 \lambda^{2} q_{\lambda}+6 \kappa^{2} q_{\kappa}\right] \ln \left(\frac{G U T}{M_{\text {mess }}}\right) . }
\end{aligned}
$$

Expressions for the first two generations can be obtained by setting $y_{t}=y_{b}=0$.

- The deflected anomaly mediation part

$$
\begin{aligned}
\delta_{\tilde{Q}_{L ; 1,2}}^{d}= & \frac{F_{\phi}^{2}}{16 \pi^{2}}\left[\frac{8}{3} G_{3} \alpha_{3}^{2}+\frac{3}{2} G_{2} \alpha_{2}^{2}+\frac{1}{30} G_{1} \alpha_{1}^{2}\right], \\
\delta_{\tilde{U}_{L ; 1,2}^{c}}^{d}= & \frac{F_{\phi}^{2}}{16 \pi^{2}}\left[\frac{8}{3} G_{3} \alpha_{3}^{2}+\frac{8}{15} G_{1} \alpha_{1}^{2}\right] \\
\delta_{\tilde{D}_{L ; 1,2,3}^{c}}^{d}= & \frac{F_{\phi}^{2}}{16 \pi^{2}}\left[\frac{8}{3} G_{3} \alpha_{3}^{2}+\frac{2}{15} G_{1} \alpha_{1}^{2}\right] \\
\delta_{\tilde{L}_{L ; 1,2,3}}^{d}= & \frac{F_{\phi}^{2}}{16 \pi^{2}}\left[\frac{3}{2} G_{2} \alpha_{2}^{2}+\frac{3}{10} G_{1} \alpha_{1}^{2}\right] \\
\delta_{\tilde{E}_{L ; 1,2,3}^{c}}^{d}= & \frac{F_{\phi}^{2}}{16 \pi^{2}} \frac{6}{5} G_{1} \alpha_{1}^{2} \\
\delta_{H_{u}}^{d}= & \frac{F_{\phi}^{2}}{16 \pi^{2}}\left[\frac{3}{2} G_{2} \alpha_{2}^{2}+\frac{3}{10} G_{1} \alpha_{1}^{2}\right] \\
& +\frac{F_{\phi}^{2}}{\left(16 \pi^{2}\right)^{2}}\left[\lambda^{2} G_{\lambda}+3 y_{t}^{2} G_{y_{t}}\right] \\
& -2 d \frac{F_{\phi}^{2}}{\left(16 \pi^{2}\right)^{2}} \lambda^{2}\left[3\left(\lambda_{P}^{T}\right)^{2}+2\left(\lambda_{P}^{D}\right)^{2}\right] \\
\delta_{H_{d}}^{d}= & \frac{F_{\phi}^{2}}{16 \pi^{2}}\left[\frac{3}{2} G_{2} \alpha_{2}^{2}+\frac{3}{10} G_{1} \alpha_{1}^{2}\right] \\
& +\frac{F_{\phi}^{2}}{\left(16 \pi^{2}\right)^{2}}\left[\lambda^{2} G_{\lambda}+3 y_{b}^{2} G_{y_{b}}\right] \\
& -2 d \frac{F_{\phi}^{2}}{\left(16 \pi^{2}\right)^{2}} \lambda^{2}\left[3\left(\lambda_{P}^{T}\right)^{2}+2\left(\lambda_{P}^{D}\right)^{2}\right] \\
&
\end{aligned}
$$

with

$$
\begin{aligned}
G_{y_{t}} & =6 y_{t}^{2}+y_{b}^{2}+\lambda^{2}-\frac{16}{3} g_{3}^{2}-3 g_{2}^{2}-\frac{13}{15} g_{1}^{2}, \\
G_{y_{b}} & =y_{t}^{2}+6 y_{b}^{2}+\lambda^{2}-\frac{16}{3} g_{3}^{2}-3 g_{2}^{2}-\frac{7}{15} g_{1}^{2}, \\
G_{\lambda} & =4 \lambda^{2}+2 \kappa^{2}+3 y_{t}^{2}+3 y_{b}^{2}-3 g_{2}^{2}-\frac{3}{5} g_{1}^{2}, \\
G_{\kappa} & =6 \lambda^{2}+6 \kappa^{2},
\end{aligned}
$$

and $N=2$ in our scenario for

$$
\begin{aligned}
G_{i} & =N d^{2}+2 N d-b_{i}, \\
\left(b_{1}, b_{2}, b_{3}\right) & =\left(\frac{33}{5}, 1,-3\right) .
\end{aligned}
$$

For the third generation $\tilde{Q}_{L, 3}, \tilde{U}_{L}^{c}$, we need to include the ' $y_{t}, y_{b}$ ' Yukawa contributions

$\delta_{\tilde{Q}_{L, 3}}^{d}=\delta_{\tilde{Q}_{L ; 1,2}}^{d}+F_{\phi}^{2} \frac{1}{\left(16 \pi^{2}\right)^{2}}\left[y_{t}^{2} G_{y_{t}}+y_{b}^{2} G_{y_{b}}\right]$, 


$$
\begin{aligned}
\delta_{\tilde{U}_{L, 3}^{c}}^{d} & =\delta_{\tilde{U}_{L ; 1,2}^{c}}^{d}+F_{\phi}^{2} \frac{1}{\left(16 \pi^{2}\right)^{2}} 2 y_{t}^{2} G_{y_{t}}, \\
\delta_{\tilde{D}_{L, 3}^{c}}^{d} & =\delta_{\tilde{D}_{L ; 1,2}^{c}}^{d}+F_{\phi}^{2} \frac{1}{\left(16 \pi^{2}\right)^{2}} 2 y_{b}^{2} G_{y_{b}} .
\end{aligned}
$$

The contributions to $\delta_{\tilde{S}}^{I}$ can be divided into three parts

$\delta_{\tilde{S}}^{d}=\Delta_{P}^{A}+\Delta_{P}^{G}+\Delta_{P}^{I}$.

We have the pure anomaly mediation part

$$
\Delta_{P}^{A}=\frac{F_{\phi}^{2}}{\left(16 \pi^{2}\right)^{2}}\left[2 \lambda^{2} G_{\lambda}+2 \kappa^{2} G_{\kappa}\right]
$$

Besides, the $m_{\tilde{S}}^{2}$ term receives new contributions involving $\lambda_{P}$ because $G_{S}$ is not continuous across the messenger threshold

$$
\Delta G_{S}=-\frac{1}{8 \pi^{2}}\left[3\left(\lambda_{P}^{T}\right)^{2} Z_{X X}^{-1} Z_{\bar{X} \bar{X}}^{-1}+2\left(\lambda_{P}^{D}\right)^{2} Z_{Y Y}^{-1} Z_{\bar{Y} \bar{Y}}^{-1}\right] .
$$

So the Yukawa mediation contribution is

$$
\begin{aligned}
\Delta_{P}^{G}= & -\frac{d^{2} F_{\phi}^{2}}{4\left(8 \pi^{2}\right)}\left[3\left(\lambda_{P}^{T}\right)^{2}\left(G_{\lambda_{P}^{T}}^{+}\right)+2\left(\lambda_{P}^{D}\right)^{2}\left(G_{\lambda_{P}^{D}}^{+}\right)\right] \\
& +\frac{d^{2} F_{\phi}^{2}}{16 \pi^{2}}\left(\lambda^{2} \Delta G_{\lambda}+\kappa^{2} \Delta G_{\kappa}\right),
\end{aligned}
$$

with

$$
\begin{aligned}
G_{\lambda_{P}^{T}}^{+}= & -\frac{1}{8 \pi^{2}}\left[5\left(\lambda_{P}^{T}\right)^{2}+2\left(\lambda_{P}^{D}\right)^{2}+2 \lambda^{2}+2 \kappa^{2}\right. \\
& \left.+2\left(\lambda_{X}^{T}\right)^{2}-\left(\frac{16}{3} g_{3}^{2}+\frac{4}{15} g_{1}^{2}\right)\right], \\
G_{\lambda_{P}^{D}}^{+}= & -\frac{1}{8 \pi^{2}}\left[3\left(\lambda_{P}^{T}\right)^{2}+4\left(\lambda_{P}^{D}\right)^{2}+2 \lambda^{2}+2 \kappa^{2}\right. \\
& \left.+2\left(\lambda_{X}^{D}\right)^{2}-\left(3 g_{2}^{2}+\frac{3}{5} g_{1}^{2}\right)\right], \\
\Delta G_{\kappa}= & -\frac{1}{8 \pi^{2}} 3\left[3\left(\lambda_{P}^{T}\right)^{2}+2\left(\lambda_{P}^{D}\right)^{2}\right], \\
\Delta G_{\lambda}= & -\frac{1}{8 \pi^{2}}\left[3\left(\lambda_{P}^{T}\right)^{2}+2\left(\lambda_{P}^{D}\right)^{2}\right] .
\end{aligned}
$$

The anomaly-gauge (Yukawa) mixing term is given by

$$
\begin{aligned}
\Delta_{P}^{I}= & -\frac{2 d F_{\phi}^{2}}{\left(16 \pi^{2}\right)^{2}}\left\{2 \lambda^{2}\left[3\left(\lambda_{P}^{T}\right)^{2}+2\left(\lambda_{P}^{D}\right)^{2}\right]\right. \\
& \left.+6 \kappa^{2}\left[3\left(\lambda_{P}^{T}\right)^{2}+2\left(\lambda_{P}^{D}\right)^{2}\right]\right\} .
\end{aligned}
$$

- The interference terms involving the Kahler modulus ' $T$ ':

$$
\begin{aligned}
\delta_{\tilde{Q}_{L}}^{I}= & \frac{M_{0} F_{\phi}}{8 \pi^{2}}\left[y_{t}^{2}\left(q_{y_{t}}-\frac{1}{8 \pi^{2}} K_{y_{t}}\right)\right. \\
& +y_{b}^{2}\left(q_{y_{b}}-\frac{1}{8 \pi^{2}} K_{y_{b}}\right) \\
& -\left(\frac{8}{3} \frac{g_{3}^{4}}{g_{3}^{2}(G U T)}+\frac{3}{2} \frac{g_{2}^{4}}{g_{2}^{2}(G U T)}\right. \\
& \left.\left.+\frac{1}{30} \frac{g_{1}^{4}}{g_{1}^{2}(G U T)}\right)\right], \\
\delta_{\tilde{U}_{L}^{c}}^{I}= & \frac{M_{0} F_{\phi}}{8 \pi^{2}}\left[2 y_{t}^{2}\left(q_{y_{t}}-\frac{1}{8 \pi^{2}} K_{y_{t}}\right)\right. \\
& \left.-\left(\frac{8}{3} \frac{g_{3}^{4}}{g_{3}^{2}(G U T)}+\frac{8}{15} \frac{g_{1}^{4}}{g_{1}^{2}(G U T)}\right)\right], \\
\delta_{\tilde{D}_{L}^{c}}^{I}= & \frac{M_{0} F_{\phi}}{8 \pi^{2}}\left[2 y_{b}^{2}\left(q_{y_{b}}-\frac{1}{8 \pi^{2}} K_{y_{b}}\right)\right. \\
& \left.-\left(\frac{8}{3} \frac{g_{3}^{4}}{g_{3}^{2}(G U T)}+\frac{2}{15} \frac{g_{1}^{4}}{g_{1}^{2}(G U T)}\right)\right],
\end{aligned}
$$

$$
\delta_{\tilde{L}_{L}}^{I}=-\frac{M_{0} F_{\phi}}{8 \pi^{2}}\left(\frac{3}{2} \frac{g_{2}^{4}}{g_{2}^{2}(G U T)}+\frac{3}{10} \frac{g_{1}^{4}}{g_{1}^{2}(G U T)}\right),
$$$$
\delta_{\tilde{E}_{L}^{c}}^{I}=-\frac{M_{0} F_{\phi}}{8 \pi^{2}}\left(\frac{6}{5} \frac{g_{1}^{4}}{g_{1}^{2}(G U T)}\right),
$$

$$
\begin{aligned}
\delta_{H_{u}}^{I}= & \frac{M_{0} F_{\phi}}{8 \pi^{2}}\left[3 y_{t}^{2}\left(q_{y_{t}}-\frac{1}{8 \pi^{2}} K_{y_{t}}\right)\right. \\
& +\lambda^{2}\left(q_{\lambda}-\frac{1}{8 \pi^{2}} K_{\lambda}\right) \\
& \left.-\left(\frac{3}{2} \frac{g_{2}^{4}}{g_{2}^{2}(G U T)}+\frac{3}{10} \frac{g_{1}^{4}}{g_{1}^{2}(G U T)}\right)\right], \\
\delta_{H_{d}}^{I}= & \frac{M_{0} F_{\phi}}{8 \pi^{2}}\left[3 y_{b}^{2}\left(q_{y_{b}}-\frac{1}{8 \pi^{2}} K_{y_{b}}\right)\right. \\
& +\lambda^{2}\left(q_{\lambda}-\frac{1}{8 \pi^{2}} K_{\lambda}\right) \\
& \left.-\left(\frac{3}{2} \frac{g_{2}^{4}}{g_{2}^{2}(G U T)}+\frac{3}{10} \frac{g_{1}^{4}}{g_{1}^{2}(G U T)}\right)\right], \\
\delta_{\tilde{S}}^{I}= & \frac{M_{0} F_{\phi}}{8 \pi^{2}}\left[2 \lambda^{2}\left(q_{\lambda}-\frac{1}{8 \pi^{2}} K_{\lambda}\right)\right. \\
& \left.+2 \kappa^{2}\left(q_{\kappa}-\frac{1}{8 \pi^{2}} K_{\kappa}\right)\right]+\Delta_{P}^{T X}\left(m_{\tilde{S}^{2}}\right) .
\end{aligned}
$$

Note that the expressions for sfermions are hold for the third generation, the first two generation can be obtained 
by setting $y_{t}, y_{b} \rightarrow 0$. Within the expressions, modular weight $l_{i}=1$ for gauge couplings are used.

The previous contributions are just the anomaly-modulus interference part. Possible modulus-gauge interference part will also appear in our scenario. The anomalous dimensions for all fields except $S$ are continuous across the messenger threshold, so their modulus-gauge interference contributions vanish. As noted previously, the anomalous dimension for $S$ is discontinuous across the messenger threshold, so we have the new $T, X$ interference contributions to $m_{\tilde{S}}^{2}$

$$
\begin{aligned}
\Delta_{P}^{T X}\left(m_{\tilde{S}^{2}}\right)= & -\frac{d M_{0} F_{\phi}}{8 \pi^{2}}\left[3\left(\lambda_{P}^{T}\right)^{2}\left(q_{\lambda_{P}^{T}}-\frac{1}{8 \pi^{2}} K_{\lambda_{P}^{T}}\right)\right. \\
& \left.+2\left(\lambda_{P}^{D}\right)^{2}\left(q_{\lambda_{P}^{D}}-\frac{1}{8 \pi^{2}} K_{\lambda_{P}^{D}}\right)\right],
\end{aligned}
$$

with

$$
\begin{aligned}
K_{\lambda_{P}^{T}}= & {\left[5\left(\lambda_{P}^{T}\right)^{2} q_{\lambda_{P}^{T}}+2\left(\lambda_{P}^{D}\right)^{2} q_{\lambda_{P}^{D}}+2 \lambda^{2} q_{\lambda}+2 \kappa^{2} q_{\kappa}\right.} \\
& \left.+2\left(\lambda_{X}^{T}\right)^{2} q_{\lambda_{X}^{T}}-\left(\frac{16}{3} g_{3}^{2}+\frac{4}{15} g_{1}^{2}\right)\right] \ln \left(\frac{G U T}{M_{\text {mess }}}\right), \\
K_{\lambda_{P}^{D}}= & {\left[3\left(\lambda_{P}^{T}\right)^{2} q_{\lambda_{P}^{T}}+4\left(\lambda_{P}^{D}\right)^{2} q_{\lambda_{P}^{D}}+2 \lambda^{2} q_{\lambda}+2 \kappa^{2} q_{\kappa}\right.} \\
& \left.+2\left(\lambda_{X}^{D}\right)^{2} q_{\lambda_{X}^{D}}-\left(3 g_{2}^{2}+\frac{3}{5} g_{1}^{2}\right)\right] \ln \left(\frac{G U T}{M_{\text {mess }}}\right) .
\end{aligned}
$$

Here

$$
\begin{aligned}
& q_{\lambda_{P}^{T}}=m_{S}+m_{\tilde{X}_{1}}+m_{X_{2}}, \\
& q_{\lambda_{P}^{D}}=m_{S}+m_{\tilde{Y}_{1}}+m_{Y_{2}}, \\
& q_{\lambda_{X}^{T}}=m_{X}+m_{\tilde{X}_{m}}+m_{X_{m}}, \\
& q_{\lambda_{X}^{D}}=m_{X}+m_{\tilde{Y}_{m}}+m_{Y_{m}} .
\end{aligned}
$$

\section{Numerical results}

After fixing the modular weights, the remaining free parameters in our scenario are

$d, \alpha, M_{m e s s}, M_{0}, \lambda, \kappa, \lambda_{P}^{D}, \lambda_{P}^{T}, \lambda_{X}^{D}, \lambda_{X}^{T}$

with $F_{\phi} /\left(16 \pi^{2}\right) \approx \alpha M_{0}$ and the simplest choice $\lambda_{P}^{D}=\lambda_{P}^{T}=$ $\lambda_{X}^{D}=\lambda_{X}^{T}=\lambda_{0}$ in our numerical study. Note that for later convenience, the definition of $\alpha$ is four times smaller than $\alpha^{\prime}$ that appears in Eq. (2.8). The ratio $\alpha$ between $F_{\phi} /\left(16 \pi^{2}\right)$ and $M_{0}$ holds in the messenger scale and in general is different from its value at the GUT scale. We choose a positive $\alpha$ in our numerical study. For a negative $\alpha$, virtual mirage unification at a super-GUT energy scale will appear.
We need to check if successful EWSB conditions are indeed fulfilled. In fact, the soft SUSY mass $m_{H_{u}}^{2}, m_{H_{d}}^{2}, m_{S}^{2}$ can be reformulated into $\mu, \tan \beta, M_{Z}^{2}$ by the minimum conditions of the scalar potential. Usually, $M_{A}$ can be used to replace $A_{\kappa}$

$$
M_{A}^{2}=\frac{2 \mu_{e f f}}{\sin 2 \beta} B_{e f f}, \quad \mu_{e f f} \equiv \lambda\langle s\rangle, \quad B_{e f f}=\left(A_{\lambda}+\kappa\langle s\rangle\right) .
$$

In order to transform $m_{H_{u}}^{2}, m_{H_{d}}^{2}, m_{S}^{2}$ into $\mu, \tan \beta, M_{Z}^{2}$, we use the following approximation

$$
\begin{aligned}
\left|\mu_{e f f}\right|^{2}= & -\frac{M_{Z}^{2}}{2}-m_{H_{u}}^{2} \\
& +\frac{1}{\tan ^{2} \beta}\left(m_{H_{d}}^{2}-m_{H_{u}}^{2}\right)+\mathcal{O}\left(1 / \tan ^{4} \beta\right), \\
\sin 2 \beta= & \frac{2 B_{e f f} \mu_{e f f}}{m_{H_{u}}^{2}+m_{H_{d}}^{2}+2\left|\mu_{e f f}\right|^{2}+\lambda^{2} v^{2}},
\end{aligned}
$$

to obtain $\mu$ and $\tan \beta$ iteratively.

We use NMSSMTools5.2.0 $[55,56]$ to scan the whole parameter space. The parameters are chosen to satisfy:

$$
\begin{aligned}
& 10^{15} \mathrm{GeV}>M_{\text {mess }}>10^{5} \mathrm{GeV}, \\
& 100 \mathrm{TeV}>M_{0}>0.1 \mathrm{TeV}, \\
& 16>\alpha>0, \quad 5>d>0, \quad 0.7>\lambda, \kappa>0, \\
& \sqrt{4 \pi}>\lambda_{0}>0 .
\end{aligned}
$$

In our scan, we impose the following constraints:

(I) The conservative lower bounds on SUSY particles [5760] from the LHC

- Gluino mass: $m_{\tilde{g}} \gtrsim 1.8 \mathrm{TeV}$.

- Light stop mass: $m_{\tilde{t}_{1}} \gtrsim 0.85 \mathrm{TeV}$.

- Light sbottom mass $m_{\tilde{b}_{1}} \gtrsim 0.84 \mathrm{TeV}$.

- Degenerated first two generation squarks $m_{\tilde{q}} \gtrsim$ $1.0 \sim 1.4 \mathrm{TeV}$.

(II) The CP-even component $S_{2}$ in the Goldstone-'eaten' combination of $H_{u}$ and $H_{d}$ doublets corresponds to the SM Higgs. The $S_{2}$ dominated CP-even scalar should lie in the combined mass range for the Higgs boson: $122 \mathrm{GeV}<M_{h}<128 \mathrm{GeV}$ from ATLAS and CMS data $[4,5]$. Note that the uncertainty is $3 \mathrm{GeV}$ instead of the default $2 \mathrm{GeV}$ because a large $\lambda$ may induce an additional $1 \mathrm{GeV}$ correction to $m_{h}$ at two-loop level [61], which is not included in the NMSSMTools.

(III) The relic density of the neutralino dark matter should satisfy the Planck data $\Omega_{D M}=0.1199 \pm 0.0027$ [62] in combination with the WMAP data [63] (with a $10 \%$ theoretical uncertainty).

(IV) The electroweak precision observables [64] and the lower bounds on neutralinos and charginos, including 
the invisible decay bounds for $Z$-boson. The most stringent constraints of LEP require $m_{\tilde{\chi}^{ \pm}}>103.5 \mathrm{GeV}$ and the invisible decay width $\Gamma\left(Z \rightarrow \tilde{\chi}_{0} \tilde{\chi}_{0}\right)<1.71 \mathrm{MeV}$, which is consistent with the $2 \sigma$ precision EW measurement $\Gamma_{i n v}^{n o n-S M}<2.0 \mathrm{MeV}$ [65].

(V) Flavor constraints [66] from B-meson rare decays

$$
\begin{aligned}
& 1.7 \times 10^{-9}<\operatorname{Br}\left(B_{S} \rightarrow \mu^{+} \mu^{-}\right)<4.5 \times 10^{-9} \\
& 0.85 \times 10^{-4}<\operatorname{Br}\left(B^{+} \rightarrow \tau^{+} \nu\right)<2.89 \times 10^{-4}, \\
& 2.99 \times 10^{-4}<\operatorname{Br}\left(B_{S} \rightarrow X_{s} \gamma\right)<3.87 \times 10^{-4} .
\end{aligned}
$$

(VI) The tension between the theoretical prediction and the experimental value for the muon anomalous magnetic moment should be ameliorated by additional positive SUSY contributions. The E821 experimental result for the muon $g-2$ at the Brookhaven AGS [67] was given by

$a_{\mu}^{\text {expt }}=116592089(63) \times 10^{-11}$,

which is larger than the SM prediction $[68,69]$

$a_{\mu}^{\mathrm{SM}}=116591834(49) \times 10^{-11}$.

The deviation is about $3 \sigma$

$$
\Delta a_{\mu}(\operatorname{expt}-\mathrm{SM})=(255 \pm 80) \times 10^{-11} .
$$

We adopt a conservative estimation $4.7 \times 10^{-10} \lesssim$ $\Delta a_{\mu} \lesssim 52.7 \times 10^{-10}$ in our numerical results.

We should note that the numerical results depend crucially on whether the $125 \mathrm{GeV}$ Higgs is the lightest CP-even scalar (Type A) or the second lightest CP-even scalar (Type B). We have the following discussions

- The low energy soft SUSY breaking spectrum of NMSSM, determined from a top-down approach by a UV-completed theory, is always bothered by the requirement to achieve successful EWSB. As noted previously, EWSB conditions in NMSSM in general require a large VEV for the singlet and consequently prefer a negative $m_{S}^{2}$ and/or large $A_{\lambda}, A_{\kappa}$ for the singlet potential. However, ordinary mirage mediation scenarios always predict large positive values for $m_{S}^{2}$ and not very large $A_{\lambda}, A_{\kappa}$, suppressing the singlet VEV. In our scenario, because of possible negative contributions to $m_{S}^{2}$ from new Yukawa interactions, stringent constraints from successful EWSB can be ameliorated. The observed $125 \mathrm{GeV}$ Higgs mass, whether it is the lightest or the second-lightest $\mathrm{CP}$-even scalar in
NMSSM, can also be successfully accommodated in our scenario.

We can see from Fig. 1 that many samples of $(\lambda, \kappa)$ can survive the EWSB conditions in NMSSM. In contrast to the numerical results of $\mathrm{TeV}$ mirage mediation in $[36,37]$ within which the allowed $(\lambda, \kappa)$ only take values near $(0.7,0.1)$, some portion of $(\lambda, \kappa)$ parameter space can survive all constraints in Type $\mathbf{A}$.

We should note that such a difference has the following reasons

- The choices of the modular weights in [36,37], for example, the values of $m_{Q_{L}^{3}}$ and $m_{t_{L}^{c}}$ etc, are different to ours which are given in Eq. (3.9).

- New ingredients, such as the messenger sector which can cause additional deflection of the RGE trajectory, will introduce new free parameters. After all, the mirage mediation scenario can be seen as a special case of our scenario with gauge couplings (Yukawa couplings) being switched off.

- The mirage unification scale is set to lie at $\mathrm{TeV}$ scale in [36,37]. It is known that simple mirage unification for soft parameters would in general be spoiled in deflected mirage mediation scenario. However, 'mirage' unification for gaugino masses persists which can be proven in our Wilsonian approach (see Appendix A for details).

In our scenario, the mirage unification scale for gaugino masses is not constrained to lie at $\mathrm{TeV}$ scale. However, we can see from the mirage unification scales shown by different colors in Fig. 1 that even if such scales are required to lie at $\mathrm{TeV}$ scale, vast parameter space, which is larger than the numerical result of Refs. ([36,37]), can survive the constraints from (I-V).

Note that the vacuum stability bounds are also taken into account in our numerical studies, which impose stringent constraints on scenario in [36,37]. In Type B in which the $125 \mathrm{GeV}$ Higgs is the second lightest CP-even scalar, the allowed $(\lambda, \kappa)$ parameter space is also much bigger than that in $[36,37]$. The Higgs mass can be increased by 8 $\mathrm{GeV}$ through the mixing with the singlet component for large $\tan \beta$ and $\lambda \lesssim 0.04$.

It can be seen from Fig. 2 that the modulus mediation contribution $M_{0}$ is bounded to lie between 1 and $8.5 \mathrm{TeV}$ for Type A. A small $M_{0}$ always prefers a low messenger scale $M_{\text {mess }}$. A large $M_{0}$, which controls the whole soft SUSY breaking parameters to be heavy, can easily accommodate the SM-like Higgs mass because of large loop corrections from heavy stops in addition to the treelevel contributions involving $\lambda$. The value of $M_{0}$ is upper bounded to be less than about $5.5 \mathrm{TeV}$ for Type $\mathbf{B}$, which sets an upper bound for the soft SUSY breaking param- 


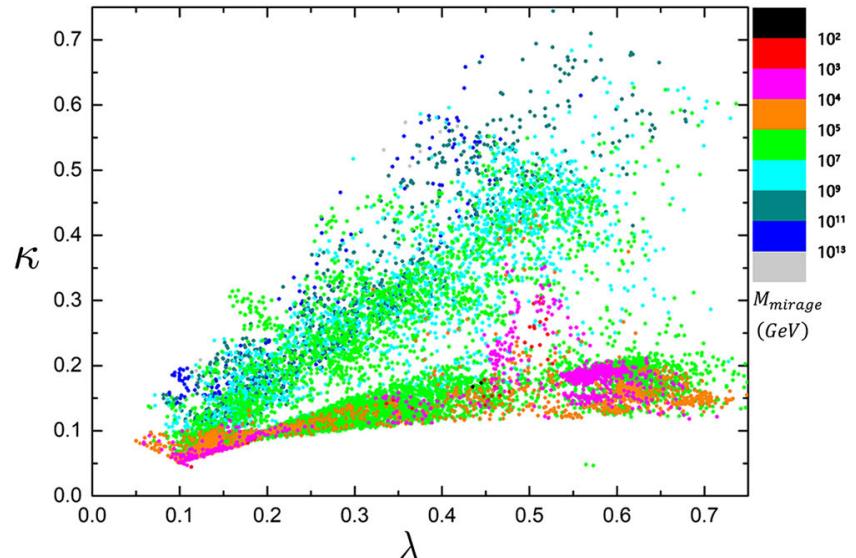

Fig. 1 The values of $(\lambda, \kappa)$ that can satisfy the EWSB conditions and at the same time accommodate the $125 \mathrm{GeV}$ Higgs boson as the lightest (left panel) or second lightest (right panel) CP-even scalar are shown.

eters, especially for the gluino masses. The gluino mass, which is determined by the scale of $M_{0}$, is bounded to below $16 \mathrm{TeV}$ for Type $\mathbf{A}$ and below $8 \mathrm{TeV}$ for Type $\mathbf{B}$, respectively.

- The Barbieri-Giudice fine-tuning (BGFT) [70] measure is defined as

$$
\Delta_{B G} \equiv \max _{i}\left|\frac{\partial \ln M_{Z}^{2}}{\partial \ln a_{i}}\right|,
$$

where ' $a_{i}$ ' stands for the set of parameters defined at the input scale.

There are two mass scales for the soft SUSY parameters in our scenario, one is the scale that characterize the modulus contributions $M_{0}$ and the other is the scale that characterize the anomaly contribution $F_{\phi}$. The latter one is rewritten into a dimensionless quantity $\alpha$ by Eq. (3.13). We can also calculate the electroweak fine-tuning (EWFT) measure $\Delta_{E W}$ of the survived points defined in $[71,72]$

$\Delta_{E W} \equiv \max _{i}\left(C_{i}\right) /\left(\frac{m_{Z}^{2}}{2}\right)$

with the relevant terms (see [71,72])

$$
\begin{aligned}
& C_{H_{u}}=\left|-\frac{m_{H_{u}}^{2} \tan ^{2} \beta}{\tan ^{2} \beta-1}\right|, C_{H_{d}}=\left|\frac{m_{H_{d}}^{2}}{\tan ^{2} \beta-1}\right|, \\
& C_{\mu}=\left|-\mu_{\text {eff }}^{2}\right|, \\
& C_{\Sigma_{u}^{u}\left(\tilde{t}_{1,2}\right)}=\frac{\tan ^{2} \beta}{\tan ^{2} \beta-1} \mid \frac{3}{16 \pi^{2}} F\left(m_{\tilde{t}_{1,2}}^{2}\right) \\
& \quad \times\left[y_{t}^{2}-g_{Z}^{2} \mp \frac{y_{t}^{2} A_{t}^{2}-8 g_{Z}^{2}\left(\frac{1}{4}-\frac{2}{3} x_{w}\right) \Delta_{t}}{m_{\tilde{t}_{2}}^{2}-m_{\tilde{t}_{1}}^{2}}\right],
\end{aligned}
$$

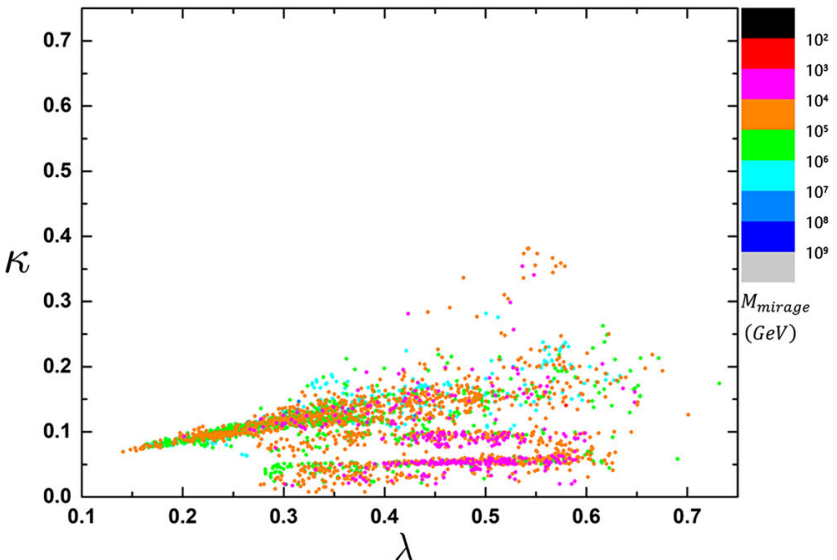

The mirage unification scales for gaugino masses are also shown in different colors. All points satisfy the constraints (I-V)

$$
\begin{aligned}
C_{\Sigma_{d}^{d}\left(\tilde{t}_{1,2}\right)} & =\frac{1}{\tan ^{2} \beta-1} \mid \frac{3}{16 \pi^{2}} F\left(m_{\tilde{t}_{1,2}}^{2}\right) \\
\times & {\left[g_{Z}^{2} \mp \frac{y_{t}^{2} \mu_{e f f}^{2}+8 g_{Z}^{2}\left(\frac{1}{4}-\frac{2}{3} x_{w}\right) \Delta_{t}}{m_{\tilde{t}_{2}}^{2}-m_{\tilde{t}_{1}}^{2}}\right] \mid, }
\end{aligned}
$$

where $x_{w}=\sin ^{2} \theta_{W}$ and

$$
\begin{aligned}
\Delta_{t} & =\frac{\left(m_{\tilde{t}_{L}}^{2}-m_{\tilde{t}_{R}}^{2}\right)}{2}+M_{Z}^{2} \cos 2 \beta\left(\frac{1}{4}-\frac{2}{3} x_{w}\right), \\
F\left(m^{2}\right) & =m^{2}\left(\log \frac{m^{2}}{m_{\tilde{t}_{1}} m_{\tilde{t}_{2}}}-1\right) .
\end{aligned}
$$

We can see from Fig. 2 that the BGFT measure in our scenario can range from $\mathcal{O}(1)$ to $\mathcal{O}(1000)$. In fact, the lowest BGFT can reach $\mathcal{O}(1)$ for Type B. Such a low fine-tuning possibly indicates that our scenario is natural. We also compare the BGFT measure of the survived points with their corresponding EWFT measure in the last two panels of Fig. 2. It can be seen that the calculated EWFT measure is positively correlated to the corresponding BGFT measure. In most of allowed parameter space, the EWFT and BGFT measures take values of the same order. Besides, the EWFT measure, which can be thought of as providing a lower bound on the electroweak fine-tuning, is always smaller than that of the BGFT measure [73].

In some of the allowed region, the EWFT measure as well as BGFT measure is rather low (being $\mathcal{O}(1)$ ). As emphasized in [73], EWFT measure is a necessary, albeit not sufficient, measure of electroweak fine-tuning. Low $\Delta_{E W}$ need not necessarily mean the model is not finetuned. Rather, it may indicate the possibility that some model might exist with low fine-tuning which might be hidden by the naive application of $\Delta_{B G}$. 

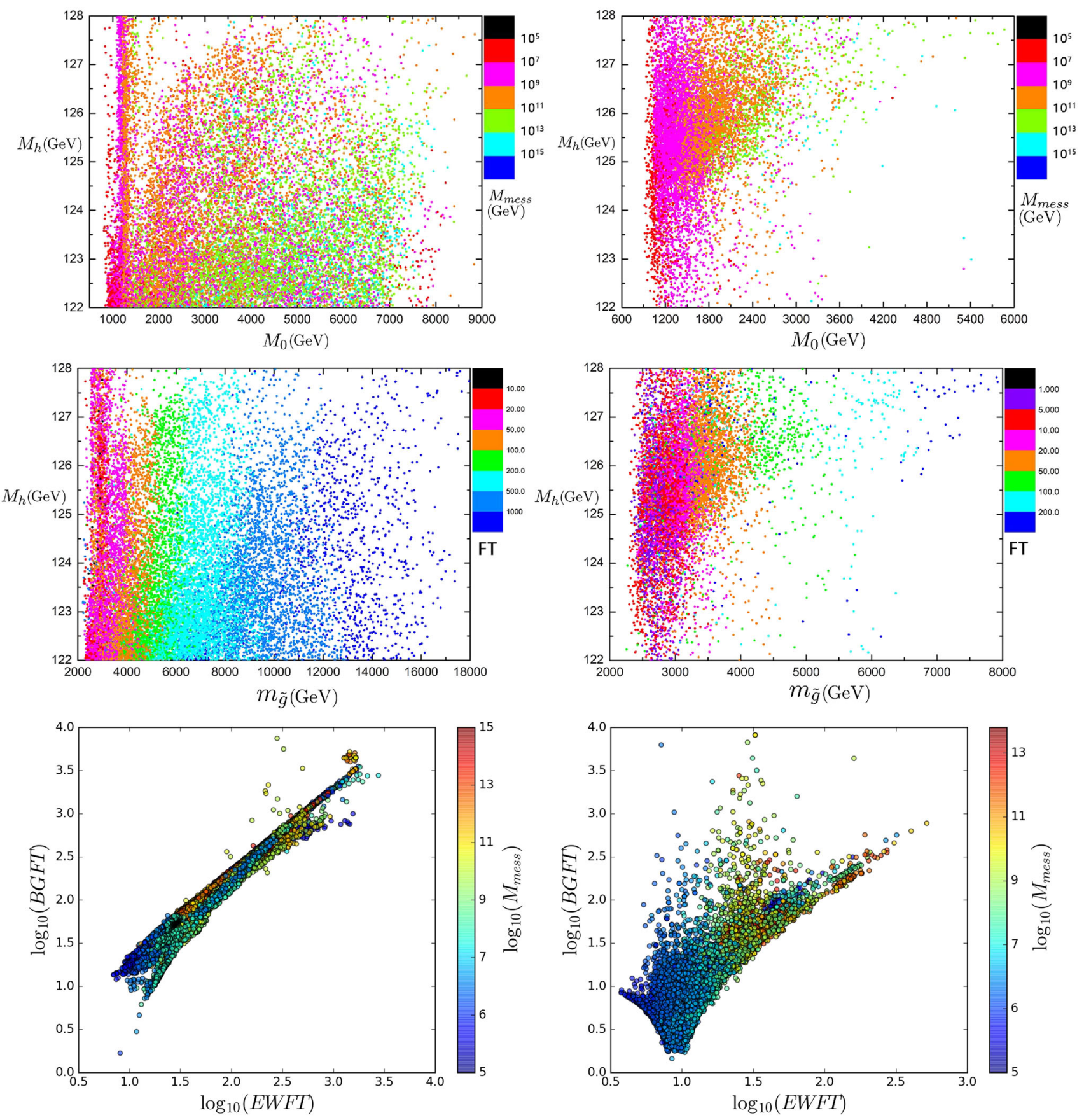

Fig. 2 Scatter plots of the SM-like Higgs mass $m_{h}$ versus the modulus mediation parameter $M_{0}$ and gluino mass $m_{\tilde{g}}$ for Type A (left panels) or Type B (right panels). The fine-tuning measures given in the middle of the panels ( $m_{h}$ vs $m_{\tilde{g}}$ ) are the Barbieri-Giudice fine tuning (BGFT)

measures. The comparisons between the BGFT measure versus the electroweak fine tuning (EWFT) measure for Type A and Type B are shown in the last two panels, respectively. All samples satisfy the constraints (I-V)

It is known that low fine-tuning needs light stops as well as a small effective $\mu$, which are naively determined by the dimensional parameter $M_{0}$ that controls the whole soft SUSY spectrum with moderate values of $\alpha$. The lower the $M_{0}$ (consequently the lower gluino mass), the lower value of the BGFT(EWFT) measure.

- A positive deflection parameter ' $d$ ' is always favored to solve the tachyonic slepton problem in the deflected AMSB for fewer messenger species. In the deflected mirage mediation scenarios, if the modulus contribution is subdominant, a realistic model still prefers a positive deflection parameter ' $d$ ' with less messenger species. As 

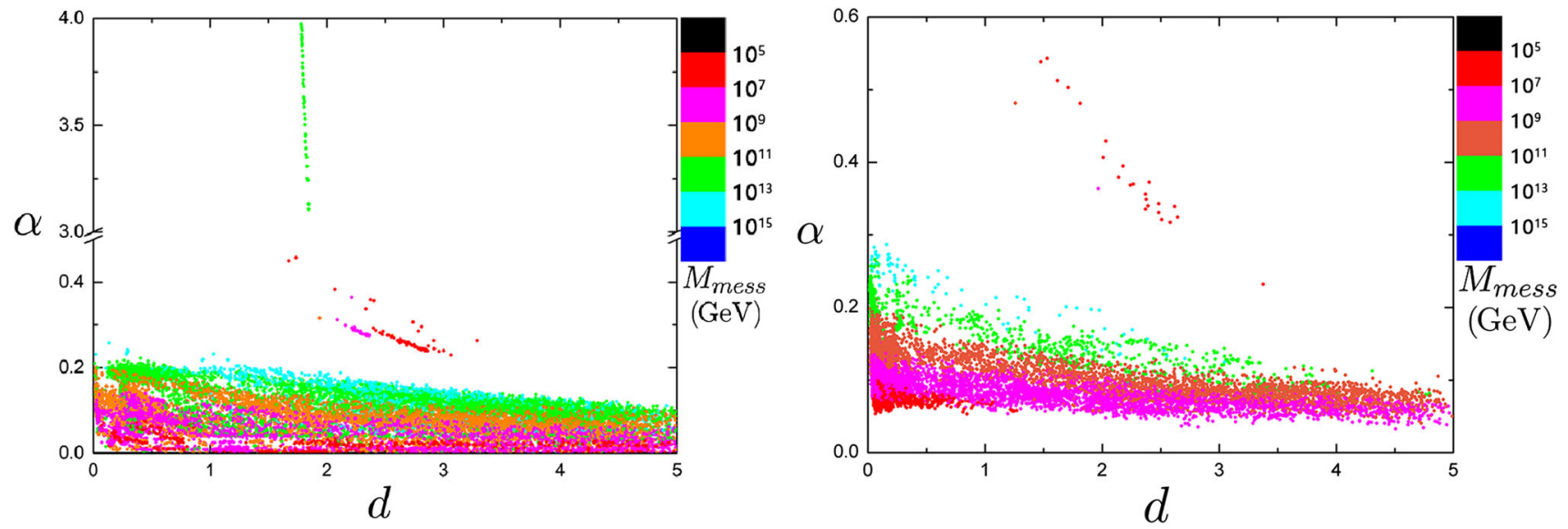

Fig. 3 The allowed regions for the deflection parameter ' $d$ ' versus $\alpha$, which parametrize the relative size between the anomaly mediation and the modulus mediation. All samples satisfy the constraints (I-V)
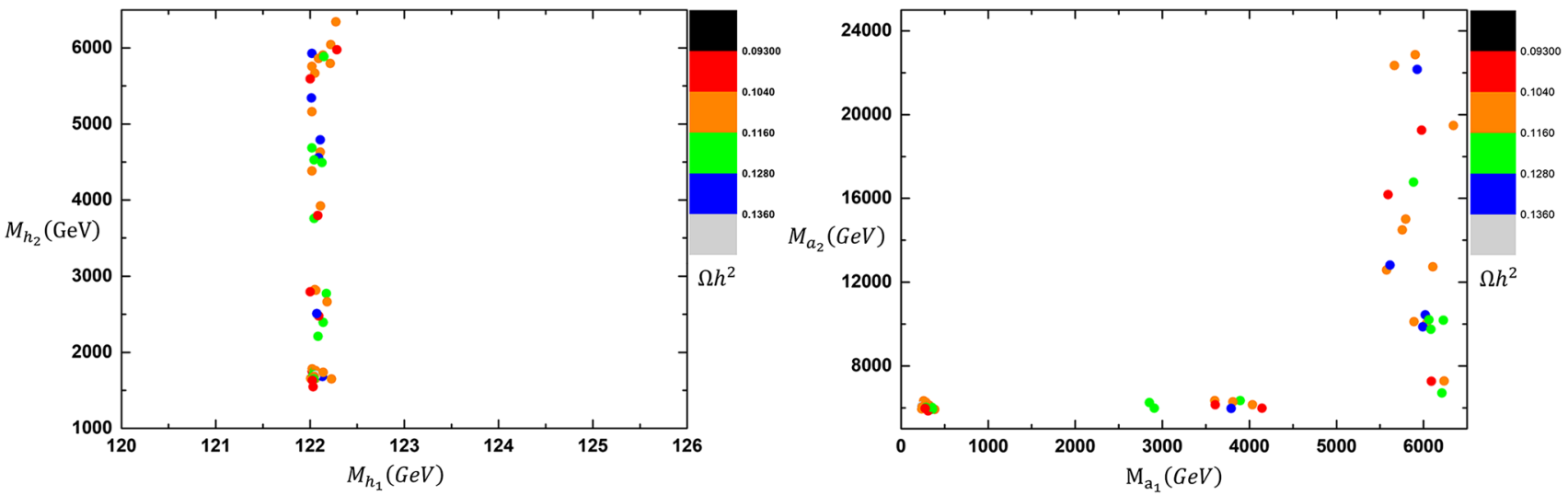

Fig. 4 The masses of the Higgs scalars with $d \approx 1.8$. All samples satisfy the constraints (I-V)

the parameter $\alpha \equiv \alpha^{\prime} / 4$ determines the relative size of the contributions between the anomaly mediation and the modulus mediation, a large value of $\alpha$, which indicates small modulus mediation contributions, needs a large positive deflection parameter ' $d$ ' to avoid tachyonic sleptons. We check that large negative values of ' $d$ ' are mostly ruled out by the EWSB condition and tachyonic sfermions. It is obvious in the left panel of Fig. 3 that the deflection parameter is constrained to lie at about 1.8 to tune the tachyonic slepton masses to positive values by additional gauge and Yukawa mediation contributions in the region with a large $\alpha$. It can be seen in Fig. 4 that the corresponding lightest CP-even Higgs mass should lie at a very narrow band centered at about $122.1 \mathrm{GeV}$ with $d \approx 1.8$. Besides, the second-lightest CP-odd scalar $a_{2}$ is constrained to lie near $6000 \mathrm{GeV}$ if the lightest CPodd scalar $a_{1}$ is lighter than $5000 \mathrm{GeV}$ while the lightest $\mathrm{CP}$-odd scalar is constrained to lie near $6000 \mathrm{GeV}$ if the second-lightest CP-odd scalar is heavier than $6000 \mathrm{GeV}$.
In our scenario, the quantity ' $4 \alpha d$ ' can approximately measure the relative size of deflection contributions (by gauge or Yukawa mediation) to the modulus mediation contributions. We can see from Fig. 3 that the deflection contributions can be dominant in a large portion of the surviving parameter space.

Besides, it can also be seen from Fig. 3 that in the modulus mediation dominated regions, that is small $\alpha$ with $d=0$, realistic NMSSM spectrums can be possible. This indicates that pure mirage mediation without deflection, which is a special case of our scenarios, can lead to realistic NMSSM spectrum even though it is stringently constrained by EWSB conditions and $125 \mathrm{GeV}$ Higgs. This conclusion agrees with that of $[36,37]$. Additional deflection with $d \neq 0$ from messenger sector can enlarge the possible choice of $\alpha$ in mirage mediation, rendering the mirage-type scenarios more natural.

- From Eq. (3.10) in the appendix, we can see the gaugino ratio at the EW scale 

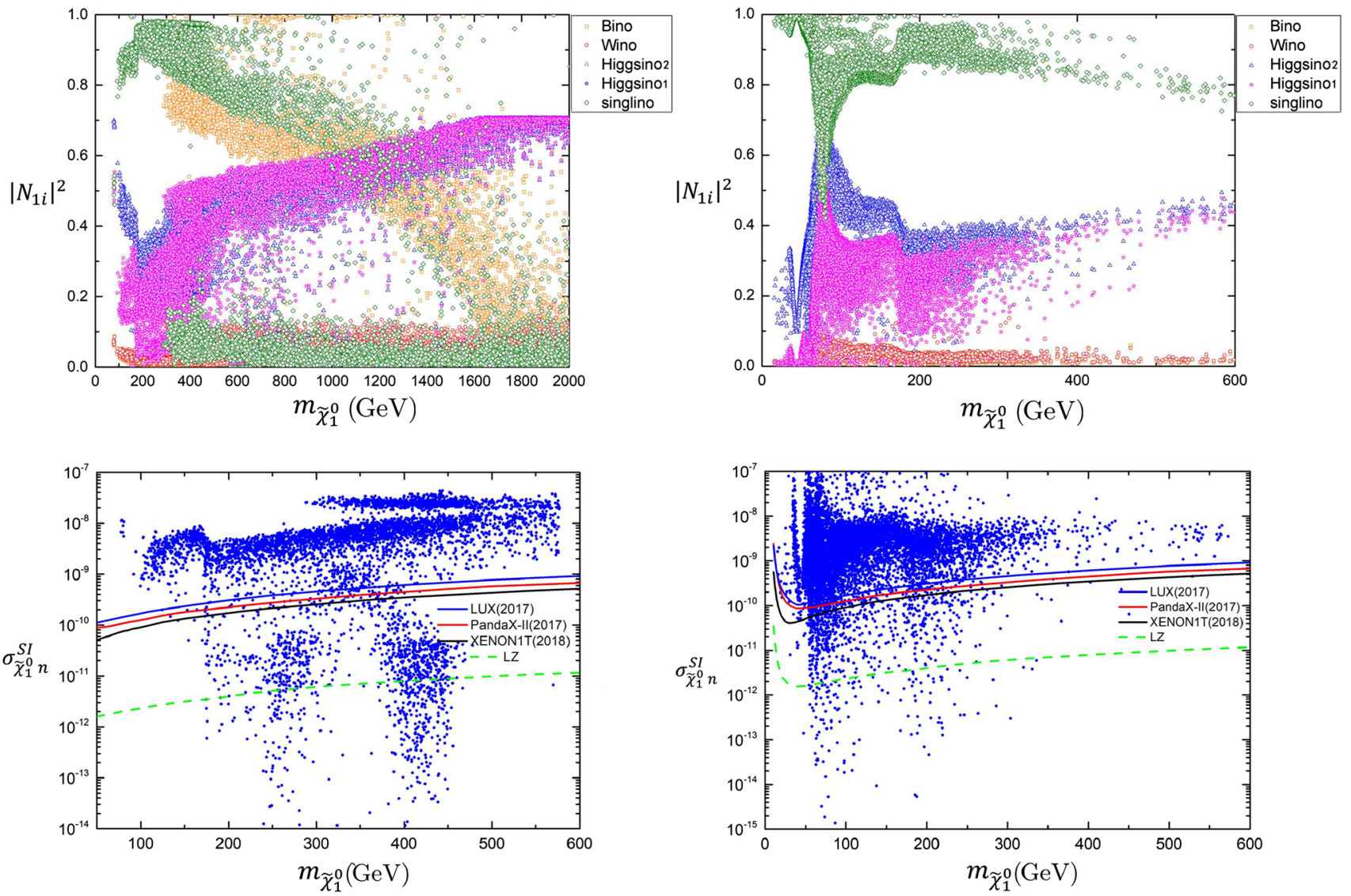

Fig. 5 The upper panels show the plots of DM mass vs the DM components for Type A (left panel) or Type B (right panel). Similarly, the lower panels show the plots of DM mass versus the spin-independent (SI) direct detection bounds. All samples satisfy the constraints from (I-V)

$$
\begin{aligned}
M_{3}: M_{2}: M_{1} \approx 6 \cdot\left[\frac{1}{g_{3}^{2}}+\alpha(-3-2 d)\right]: 2 \\
\cdot\left[\frac{1}{g_{2}^{2}}+\alpha(1-2 d)\right]:\left[\frac{1}{g_{1}^{2}}+\alpha(6.6-2 d)\right]
\end{aligned}
$$

where $g_{1}, g_{2}, g_{3}$ take values at the GUT scale. On the other hand, the singlino mass is determined by $\kappa$ and $\langle s\rangle$, which rescales the effective $\mu_{e f f}$ parameter by a factor $2 \kappa / \lambda$. In general, a pure singlino-like LSP tends to have a too large relic density due to a comparatively small annihilation cross section because of its small couplings to SM particles. Non-negligible higgsino contents within singlino-dominated neutralino DM can open several annihilation channels and be helpful to reduce the DM relic density to right amount.

In Type A, the neutralino DM is either singlino-dominant or bino-dominant, each possibility contains non-negligible higgsino components. In the bino-dominant regions, the LSP annihilate dominantly into pairs of gauge bosons $\left(W^{+} W^{-}, Z Z\right)$ and (doublet-like) Higgs bosons $\left(W^{ \pm} H^{ \pm}, Z H, H A\right.$ ) via s-channel $\mathrm{Z}$ or Higgs exchange, as well as through t-channel neutralino and chargino exchange processes. In the singlino-dominant regions, the singlino-like LSP (with the presence of non-negligible higgsino components) can annihilates via the t-channel $\chi_{1}^{0}$ exchange into pairs of mostly singlet-like $H_{1}$ and $A_{1}$ by enhanced $\chi_{1}^{0} \chi_{1}^{0} H_{1}\left(A_{1}\right)$ couplings. Co-annihilation with heavier $\chi_{2}^{0}$ (for $\Delta m \lesssim 10 \mathrm{GeV}$ ) will also efficiently reduce the singlino relic abundance to a proper $\Omega_{D M}$. Besides, the annihilation channels $\chi_{1}^{0} \chi_{1}^{0} \rightarrow t \bar{t}, b \bar{b}$ can also be important. Similar DM annihilation channels exist for Type $\mathbf{B}$ in which the neutralino DM is mainly singlinolike with non-negligible higgsino components.

We know that mixed bino-Higgsino DM is severely constrained by direct detection constraints. For a singlinodominant DM, the exchange of a light $H_{1}$ can possibly lead to a large direct detection cross section that will be accessible in the present generation of detectors. It can be seen from the lower panels of Fig. 5 that only a small portion of DM parameter space can survive the spinindependent (SI) DM direct detection constraints from the LUX [74], PANDAX [75] and Xenon1T [76]. In fact, direct detection constrained the DM mass to lie in the range $[120,470] \mathrm{GeV}$ for Type $\mathbf{A}$ and $[50,400] \mathrm{GeV}$ for Type B, respectively. 

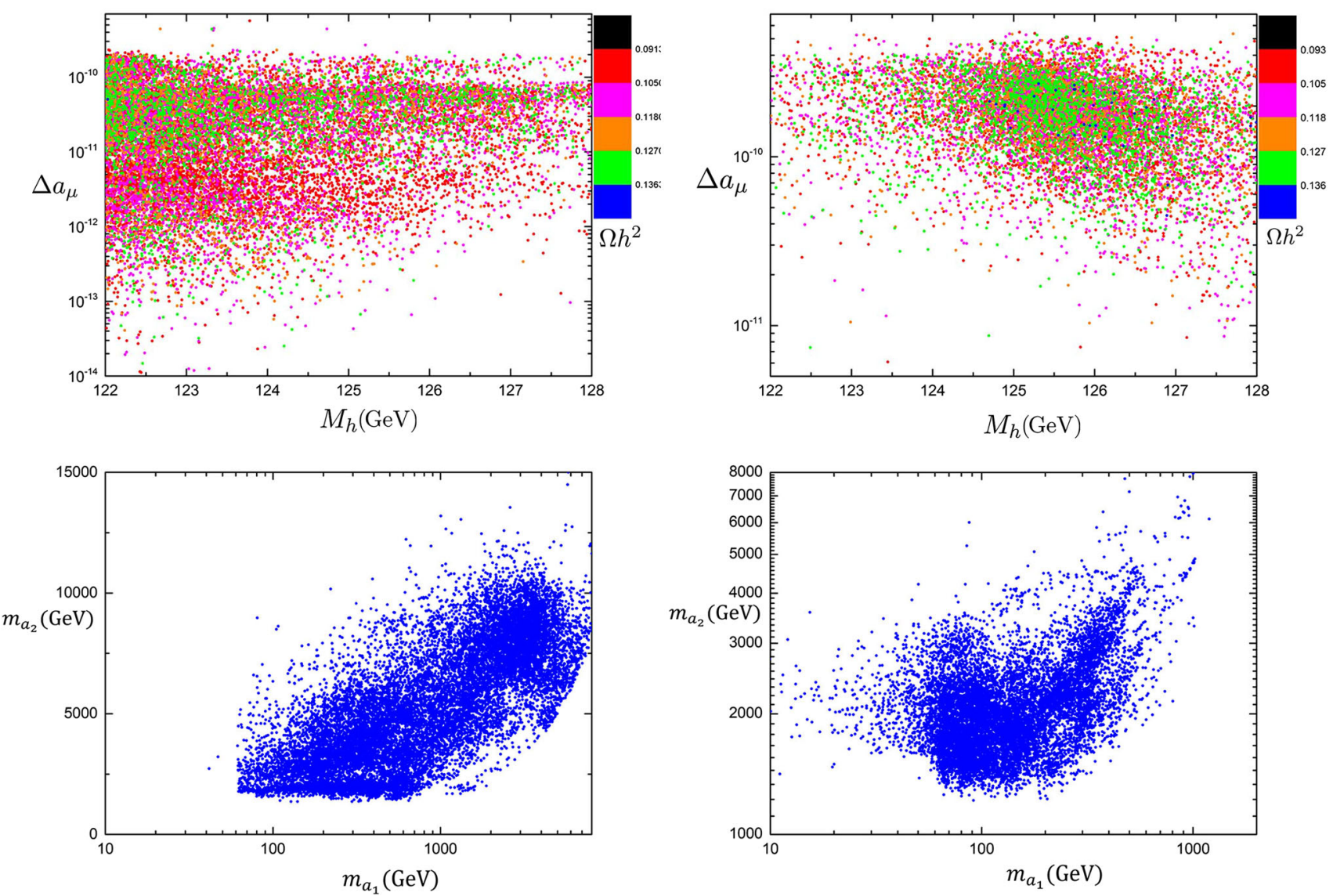

Fig. 6 The upper panels show the SUSY contributions to the muon anomalous magnetic moment $\Delta a_{\mu}$ for Type $\mathrm{A}$ (left) and Type B (right). The lower panels show the corresponding masses of the lightest $\mathrm{CP}$ -

odd scalar $m_{a_{1}}$, which can possibly give large two-loop contributions to $\Delta a_{\mu}$. All samples satisfy the constraints (I-V)

- Figure 6 shows the SUSY contributions to the muon $g-2$. It is known that the required SUSY contributions to $\Delta a_{\mu}$ can be achieved only if the relevant sparticles( $\left.\tilde{\mu}, \tilde{v}_{\mu}, \tilde{B}, \tilde{W}, \tilde{H}\right)$ are lighter than $600-700 \mathrm{GeV}$ for $\tan \beta \sim 10$ in the MSSM [77]. The inclusion of the singlino in the NMSSM can not give sizable contributions to $\Delta a_{\mu}$ because of the suppressed couplings of singlino to MSSM sector. Although the two loop contributions involving the Higgs are negligible in the SM, new Higgs bosons in the NMSSM could have an important impact on $\Delta a_{\mu}$ if the lightest neutral CP-odd Higgs scalar is very light [78]. In fact, a positive two-loop contribution is numerically more important for a light CP-odd Higgs being a bit heavier than $3 \mathrm{GeV}$ and the sum of both one-loop and two-loop contributions is maximal around $m_{a_{1}} \sim 6 \mathrm{GeV}$. In our scenario, the lightest CP-odd Higgs $a_{1}$ is bounded to be heavier than $40 \mathrm{GeV}$ and give negligible two-loop contributions to $\Delta a_{\mu}$ in Type $\mathbf{A}$. The main contribution to $\Delta a_{\mu}$ is thus similar to that in the MSSM. In Type $\mathbf{B}$, the lightest $\mathbf{C P}$-odd Higgs $a_{1}$ can lie near 10

$\mathrm{GeV}$ and will give important two-loop contributions to increase $\Delta a_{\mu}$ to values favored by experiments.

- Before we finish our discussions on numerical results, we note that the gauge-modulus interference contribution for soft scalar masses will play an important role in phenomenological studies. In our scenario, such a contribution is non-vanishing only for $S$ which is given in Eq. (3.65). This contribution will possibly change the EWSB condition, the dark matter relic density and other collider predictions. We show several benchmark points which are affected by the contributions of $\Delta_{P}^{T X}\left(m_{\tilde{S}^{2}}\right)$. Table 1 shows several benchmark points, which should not survive the various constraints if the gauge-modulus interference contribution is absent. Table 2, in contrary, shows several benchmark points which should survive the various constraints if the gauge-modulus interference contribution is absent. 
Table 1 Benchmark points, which should not survive the various constraints if the gauge-modulus interference contribution is absent. The quantities with mass dimension are in unit of GeV. Without the gaugemodulus interference contribution, the points will not survive the constraints because of the constraints shown in 'Reasons'

\begin{tabular}{|c|c|c|c|}
\hline & Sample I & Sample II & Sample III \\
\hline $\mathrm{d}$ & 0.326 & 0.257 & 0.036 \\
\hline$\alpha$ & 0.102 & 0.075 & 0.115 \\
\hline$M_{m e s s}$ & $1.420 \times 10^{9}$ & $4.823 \times 10^{6}$ & $4.724 \times 10^{7}$ \\
\hline$M_{0}$ & 1278 & 1203 & 1222 \\
\hline$\lambda$ & 0.607 & 0.430 & 0.591 \\
\hline$\kappa$ & 0.205 & 0.147 & 0.193 \\
\hline$\lambda_{0}$ & 2.389 & 1.012 & 2.597 \\
\hline$m_{\tilde{Q}_{L ; 1,2}}$ & 2041 & 1452 & 1479 \\
\hline$m_{\tilde{Q}_{L, 3}}$ & 1714 & 1081 & 1164 \\
\hline$m_{\tilde{U}_{L ; 1,2}}$ & 2096 & 1558 & 1584 \\
\hline$m_{\tilde{U}_{L, 3}^{c}}$ & 1640 & 1096 & 1236 \\
\hline$m_{\tilde{D}_{L ; 12}^{c}}$ & 2113 & 1565 & 1598 \\
\hline$m_{\tilde{D}_{L, 3}}$ & 1908 & 1322 & 1345 \\
\hline$m_{\tilde{L}_{L ; 1,2,3}}$ & 920.9 & 897.1 & 891.2 \\
\hline$m_{\tilde{E}_{L ; 1,2,3}}$ & 761.7 & 691.0 & 661.4 \\
\hline$A_{\lambda}$ & 1823 & 1523 & 1807 \\
\hline$A_{\kappa}$ & -169.2 & -163.2 & -95.60 \\
\hline$A_{t}$ & -2360 & -2821 & -2401 \\
\hline$A_{b}$ & -3481 & -3353 & -3450 \\
\hline$A_{\tau}$ & 1412 & 1453 & 1371 \\
\hline$M_{\tilde{g}}$ & 2971 & 2889 & 2775 \\
\hline$\mu_{e f f}$ & 646.3 & 200.9 & 635.2 \\
\hline$m_{h_{1}}$ & 125.8 & 125.7 & 126.9 \\
\hline$g_{\mu}-2$ & $5.049 \times 10^{-11}$ & $2.170 \times 10^{-10}$ & $6.035 \times 10^{-11}$ \\
\hline$\Omega_{\chi} h^{2}$ & 0.114 & 0.118 & 0.120 \\
\hline$m_{\tilde{\chi}_{1}^{0}}$ & 442.0 & 122.6 & 418.5 \\
\hline$\sigma_{P}^{S I}$ & $1.172 \times 10^{-11} p b$ & $2.960 \times 10^{-12} p b$ & $\begin{array}{l}1.701 \times \\
10^{-12} p b\end{array}$ \\
\hline Reasons & EWSB & EWSB & $\begin{array}{l}\text { Collider; } \\
\text { Higgs mass; } \\
\Omega h^{2}\end{array}$ \\
\hline
\end{tabular}

\section{Conclusions}

We propose to generate a realistic soft SUSY breaking spectrum for Next-to-Minimal Supersymmetric Standard Model with a generalized deflected mirage mediation scenario, in which additional Yukawa and gauge mediation contributions are included to deflect the RGE trajectory. Based on the Wilsonian effective action obtained by integrating out the messengers, the NMSSM soft SUSY breaking spectrum can be given analytically at the messenger scale. We find that additional contributions to $m_{S}^{2}$ can possibly ameliorate the
Table 2 Benchmark points, which should survive the various constraints if the gauge-modulus interference contribution is absent. The quantities with mass dimension are in unit of $\mathrm{GeV}$. With the gaugemodulus interference contribution, the points will not survive the constraints because of the constraints shown in 'Reasons'

\begin{tabular}{|c|c|c|c|}
\hline & Sample I & Sample II & Sample III \\
\hline $\mathrm{d}$ & 1.114 & 0.307 & 1.102 \\
\hline$\alpha$ & 0.056 & 0.102 & 2.804 \\
\hline$M_{m e s s}$ & $8.188 \times 10^{9}$ & $4.360 \times 10^{12}$ & $3.880 \times 10^{12}$ \\
\hline$M_{0}$ & 5049 & 2034 & 938.1 \\
\hline$\lambda$ & 0.005 & 0.097 & 0.105 \\
\hline$\kappa$ & 0.553 & 0.354 & 0.231 \\
\hline$\lambda_{0}$ & 1.597 & 2.279 & 0.381 \\
\hline$m_{\tilde{Q}_{L ; 1,2}}$ & 9087 & 3853 & 17,479 \\
\hline$m_{\tilde{Q}_{L, 3}}$ & 7478 & 3155 & 15,378 \\
\hline$m_{\tilde{U}_{L ; 1,2}}$ & 9200 & 3836 & 17,234 \\
\hline$m_{\tilde{U}_{L, 3}^{c}}$ & 6789 & 2756 & 12,746 \\
\hline$m_{\tilde{D}_{L ; 12}^{c}}$ & 9259 & 3867 & 17,223 \\
\hline$m_{\tilde{D}_{L, 3}}$ & 8366 & 3482 & 17,057 \\
\hline$m_{\tilde{L}_{L ; 1,2,3}}$ & 3648 & 1465 & 1377 \\
\hline$m_{\tilde{E}_{L ; 1,2,3}}$ & 3249 & 1449 & 3455 \\
\hline$A_{\lambda}$ & 4657 & 1511 & -2737 \\
\hline$A_{\kappa}$ & -6511 & -3614 & -4573 \\
\hline$A_{t}$ & $-10,187$ & -3843 & 9530 \\
\hline$A_{b}$ & $-11,949$ & -4828 & 6936 \\
\hline$A_{\tau}$ & 6368 & 2267 & -4347 \\
\hline$M_{\tilde{g}}$ & 11,819 & 4523 & 17,463 \\
\hline$\mu_{e f f}$ & 439.2 & 288 & 4998 \\
\hline$m_{h_{1}}$ & 127.1 & 123.8 & 123.6 \\
\hline$g_{\mu}-2$ & $1.580 \times 10^{-11}$ & $1.096 \times 10^{-10}$ & $\begin{array}{c}-1.110 \times \\
10^{-11}\end{array}$ \\
\hline$\Omega_{\chi} h^{2}$ & 0.022 & 0.010 & 0.004 \\
\hline$m_{\tilde{\chi}_{1}^{0}}$ & 458.3 & 294.5 & 421.0 \\
\hline$\sigma_{P}^{S I}$ & $1.030 \times 10^{-10} p b$ & $7.316 \times 10^{-10} p b$ & $\begin{array}{l}1.870 \times \\
10^{-12} p b\end{array}$ \\
\hline Reasons & EWSB & EWSB & $\begin{array}{l}\text { Collider; } \\
\text { Higgs mass } \\
\Omega h^{2}\end{array}$ \\
\hline
\end{tabular}

stringent constraints from the EWSB and $125 \mathrm{GeV}$ Higgs mass. Constraints from dark matter and fine-tuning are also discussed. The Barbieri-Giudice fine-tuning measure and electroweak fine-tuning measure in our scenario can be as low as $\mathcal{O}(1)$, which possibly indicates that our scenario is natural.

Acknowledgements We are very grateful to the referee for efforts to improve our paper. This work was supported by the National Natural Science Foundation of China (NNSFC) under Grant nos. 11675147, 11775012, 11705093 and 11675242, by the Young Core Instructor Foundation of Henan Education Department, by Peng-Huan-Wu The- 
oretical Physics Innovation Center (11747601), by the CAS Center for Excellence in Particle Physics (CCEPP), by the CAS Key Research Program of Frontier Sciences, by a Key R\&D Program of Ministry of Science and Technology of China under number 2017YFA040220004, and by the ARC Centre of Excellence for Particle Physics at the Tera-scale under Grant CE110001004.

Data Availability Statement This manuscript has no associated data or the data will not be deposited. [Authors' comment: The datasets generated during and/or analysed during the current study are available from the corresponding author on reasonable request.]

Open Access This article is distributed under the terms of the Creative Commons Attribution 4.0 International License (http://creativecomm ons.org/licenses/by/4.0/), which permits unrestricted use, distribution, and reproduction in any medium, provided you give appropriate credit to the original author(s) and the source, provide a link to the Creative Commons license, and indicate if changes were made. Funded by SCOAP ${ }^{3}$.

\section{Appendix A: The mirage scale in deflected mirage medi- ation mechanism}

The gaugino mass at scale $\mu$ below the messenger scale can be written as

$M_{i}=M_{0} \frac{g_{i}^{2}(\mu)}{g_{i}^{2}(G U T)}+F_{\phi} \frac{\alpha_{i}}{4 \pi}\left(b_{i}-d \Delta b_{i}\right)$,

with

$$
\begin{aligned}
\frac{1}{g_{i}^{2}(\mu)}= & \frac{1}{g_{i}^{2}(G U T)}+\frac{b_{i}+\Delta b_{i}}{8 \pi^{2}} \ln \left(\frac{M_{G}}{M}\right) \\
& +\frac{b_{i}}{8 \pi^{2}} \ln \left(\frac{M}{\mu}\right), \\
= & \frac{1}{g_{i}^{2}\left(M_{Z}\right)}-\frac{b_{i}}{8 \pi^{2}} \ln \left(\frac{\mu}{M_{Z}}\right) .
\end{aligned}
$$

Here $M_{G}, M$ denote the gauge coupling unification scale and the messenger scale, respectively. We will show that apparent 'mirage' unification for gaugino masses will still be preserved after the introduction of messenger sector in deflected mirage mediation scenarios. Substituting $\Delta b_{i} \equiv N$ and the definition $F_{\phi} \equiv\left(16 \pi^{2}\right) \alpha M_{0}$ into Eq. (A.1), the gaugino masses can rewrite as

$$
\begin{aligned}
M_{i}= & M_{0} \frac{g_{i}^{2}(\mu)}{g_{i}^{2}(G U T)}+\alpha M_{0}\left(b_{i}-d N\right) g_{i}^{2}(\mu), \\
= & {\left[M_{0}-\alpha M_{0} d N g_{i}^{2}(G U T)\right] } \\
& \times\left[1-\frac{b_{i}+N}{8 \pi^{2}} g_{i}^{2}(\mu) \ln \left(\frac{M_{G}}{M}\right)\right. \\
& \left.-\frac{b_{i}}{8 \pi^{2}} g_{i}^{2}(\mu) \ln \left(\frac{M}{\mu}\right)\right]+\alpha M_{0} b_{i} g_{i}^{2}(\mu), \\
\approx & \left(M_{0}-\alpha M_{0} d N g_{i}^{2}(G U T)\right)
\end{aligned}
$$

$$
\begin{aligned}
& \times\left[\left(1-\frac{N}{8 \pi^{2}} g_{i}^{2}\left(M_{G}\right) \ln \left(\frac{M_{G}}{M}\right)\right)\right. \\
& \left.-\frac{b_{i}}{8 \pi^{2}} g_{i}^{2}(\mu) \ln \left(\frac{M_{G}}{\mu}\right)\right]+\alpha M_{0} b_{i} g_{i}^{2}(\mu), \\
\equiv & K_{0}\left[c_{0}-\frac{b_{i}}{8 \pi^{2}} g_{i}^{2}(\mu) \ln \left(\frac{M_{G}}{\mu}\right)\right]+\alpha M_{0} b_{i} g_{i}^{2}(\mu),
\end{aligned}
$$

with

$$
\begin{aligned}
K_{0} & \equiv M_{0}-\alpha M_{0} d N g_{i}^{2}(G U T), \\
c_{0} & =1-\frac{N}{8 \pi^{2}} g_{i}^{2}\left(M_{G}\right) \ln \left(\frac{M_{G}}{M}\right) .
\end{aligned}
$$

So we can see that mirage unification for gaugino masses will be satisfied at the scale $\mu$ determined by

$$
\ln \left(\frac{M_{G}}{\mu}\right)=\frac{8 \pi^{2} \alpha M_{0}}{K_{0}} .
$$

with the mirage unification values for gaugino masses as

$$
M_{i}\left(\mu_{\text {mirage }}\right)=K_{0} c_{0} .
$$

\section{References}

1. G. Aad et al., ATLAS collaboration. Phys. Lett. B 710(2012), 67 (2011)

2. G. Aad et al., ATLAS collaboration. Phys. Rev. D 87, 012008 (2013)

3. S. Chatrchyan et al., CMS collaboration. Phys. Rev. Lett. 107, 221804 (2011)

4. G. Aad et al., ATLAS collaboration. Phys. Lett. B 710, 49 (2012)

5. S. Chatrachyan et al., CMS collaboration. Phys. Lett. B 710, 26 (2012)

6. S. Kachru, R. Kallosh, A. Linde, S.P. Trivedi, Phys. Rev. D 68, 046005 (2003)

7. K. Choi, A. Falkowski, H.P. Nilles, M. Olechowski, S. Pokorski, JHEP 0411, 076 (2004)

8. K. Choi, A. Falkowski, H.P. Nilles, M. Olechowski, Nucl. Phys. B 718, 113 (2005)

9. L. Randall, R. Sundrum, Nucl. Phys. B 557, 79 (1999)

10. G.F. Giudice, M.A. Luty, H. Murayama, R. Rattazzi, JHEP 9812, 027 (1998)

11. K. Choi, K.S. Jeong, K.I. Okumura, JHEP 0509, 039 (2005)

12. I. Jack, D.R.T. Jones, Phys. Lett. B 482, 167 (2000)

13. E. Katz, Y. Shadmi, Y. Shirman, JHEP 9908, 015 (1999)

14. N. ArkaniHamed, D.E. Kaplan, H. Murayama, Y. Nomura, JHEP 0102, 041 (2001)

15. R. Sundrum, Phys. Rev. D 71, 085003 (2005)

16. K. Hsieh, M.A. Luty, JHEP 0706, 062 (2007)

17. Y. Cai, M.A. Luty, JHEP 1012, 037 (2010)

18. T. Kobayashi, Y. Nakai, M. Sakai, JHEP 1106, 039 (2011)

19. A. Pomarol, R. Rattazzi, JHEP 9905, 013 (1999)

20. R. Rattazzi, A. Strumia, J.D. Wells, Nucl. Phys. B 576, 3 (2000)

21. N. Okada, Phys. Rev. D 65, 115009 (2002)

22. N. Okada, H.M. Tran, Phys. Rev. D 87, 035024 (2013)

23. F. Wang, W. Wang, J.M. Yang, Y. Zhang, JHEP 1507, 138 (2015). arXiv:1505.02785 [hep-ph] 
24. F. Wang, J.M. Yang, Y. Zhang, JHEP 1604, 177 (2016). arXiv:1602.01699 [hep-ph]

25. F. Wang, L. Wu, J.M. Yang, M. Zhang, Phys. Lett. B 759, 191 (2016). arXiv:1512.06715 [hep-ph]

26. M. Dine, W. Fischler, M. Srednicki, Nucl. Phys. B 189, 575 (1981)

27. S. Dimopoulos, S. Raby, Nucl. Phys. B 192, 353 (1981)

28. M. Dine, W. Fischler, Phys. Lett. B 110, 227 (1982)

29. M. Dine, A.E. Nelson, Phys. Rev. D 48, 1277 (1993)

30. M. Dine, A.E. Nelson, Y. Shirman, Phys. Rev. D 51, 1362 (1995)

31. M. Dine, A.E. Nelson, Y. Nir, Y. Shirman, Phys. Rev. D 53, 2658 (1996)

32. G.F. Giudice, R. Rattazzi, Phys. Rep. 322, 419 (1999)

33. L.L. Everett, I.-W. Kim, P. Ouyang, K.M. Zurek, Phys. Rev. Lett. 101, 101803 (2008)

34. L.L. Everett, I.-W. Kim, P. Ouyang, K.M. Zurek, JHEP 0808, 102 (2008)

35. L.L. Everett, T. Garon, B.L. Kaufman, B.D. Nelson, Phys. Rev. D 93, 055031 (2016)

36. T. Kobayashi, H. Makino, K. Okumura, T. Shimomura, T. Takahashi, JHEP 1301, 081 (2013)

37. K. Hagimoto, T. Kobayashi, H. Makino, K. Okumura, T. Shimomura, JHEP 1602, 089 (2016)

38. A. Pierce, J. Thaler, JHEP 09, 017 (2006)

39. M. Asano, T. Higaki, Phys. Rev. D 86, 035020 (2012)

40. F. Wang, JHEP 1811, 062 (2018)

41. H. Baer, V. Barger, H. Serce, X. Tata, Phys. Rev. D 94, 115017 (2016)

42. C.P. Burgess, R. Kallosh, F. Quevedo, JHEP 0310, 056 (2003)

43. A. Saltman, E. Silverstein, JHEP 0411, 066 (2004)

44. J.P. Conlon, F. Quevedo, K. Suruliz, JHEP 0508, 007 (2005)

45. K. Choi, K.S. Jeong, K. Okumura, JHEP 0807, 047 (2008)

46. A. Delgado, G.F. Giudice, P. Slavich, Phys. Lett. B 653, 424 (2007)

47. B. Altunkaynak, L.L. Everett, I. Kim, B.D. Nelson, Y. Rao, JHEP 1005, 054 (2010)

48. G.F. Giudice, R. Rattazzi, Nucl. Phys. B 511, 25 (1998)

49. J.A. Evans, D. Shih, JHEP 08, 093 (2013)

50. Z. Chacko, E. Ponton, Phys. Rev. D 66, 095004 (2002)

51. F. Wang, Phys. Lett. B 751, 402 (2015)

52. X. Ning, F. Wang, JHEP 08, 089 (2017)

53. F. Wang, W. Wang, J.M. Yang, Phys. Rev. D 96, 075025 (2017). arXiv:1703.10894 [hep-ph]

54. G.-L. Liu, F. Wang, W. Wang, J.M. Yang, Chin. Phys. C 42(3), 035101 (2018)
55. B. Allanach et al., Comp. Phys. Commun. 180, 8 (2009)

56. U. Ellwanger, C.-C. Jean-Louis, A.M. Teixeira, JHEP 0805, 044 (2008)

57. ATLAS Collboration, ATLAS-CONF-2017-020; CMS Collboration, CMS-SUS-16-051, CMSSUS-16-049

58. CMS Collboration, CMS-SUS-16-051, CMSSUS-16-049

59. ATLAS Collboration, ATLAS-CONF-2017-021; CMS Collboration, CMS-SUS-16-051

60. CMS Collboration, CMS-SUS-16-051

61. M.D. Goodsell, K. Nickel, F. Staub, Phys. Rev. D 91, 035021 (2015)

62. P.A.R. Ade et al., Planck collaboration. Astron. Astrophys. 571, A16 (2014)

63. J. Dunkley et al., WMAP Collaboration. Astrophys. J. Suppl. 180, 306 (2009)

64. C. Patrignani et al., Particle data group. Chin. Phys. C 40, 100001 (2016)

65. S. Schael et al., ALEPH and DELPHI and L3 and OPAL and SLD and LEP electroweak working group and SLD electroweak group and SLD heavy flavour group collaborations. Phys. Rep. 427, 257 (2006)

66. V. Khachatryan et al., CMS and LHCb collaborations. Nature 522, 68 (2015)

67. M. Byrne, C. Kolda, J.E. Lennon, Phys. Rev. D 67, 075004 (2003)

68. K. Hagiwara, R. Liao, A.D. Martin, D. Nomura, T. Teubner, J. Phys. G 38, 085003 (2011). arXiv: 1105.3149

69. M. Davier, A. Hoecker, B. Malaescu, Z. Zhang, Eur. Phys. J. C 71, 1515 (2011). arXiv: 1010.4180

70. R. Barbieri, G. Giudice, Nucl. Phys. B 306, 63 (1988)

71. H. Baer, V. Barger, P. Huang, A. Mustafayev, X. Tata, Phys. Rev. Lett. 109, 161802 (2012)

72. H. Baer, V. Barger, P. Huang, D. Mickelson, A. Mustafayev, X. Tata, Phys. Rev. D 87, 115028 (2013)

73. H. Baer, V. Barger, D. Mickelson, Phys. Rev. D 88, 095013 (2013)

74. D.S. Akerib et al. arXiv:1608.07648 [astro-ph.CO]

75. C. Fu et al., Phys. Rev. Lett. 118, 071301 (2017). arXiv:1611.06553

76. E. Aprile, et al. [XENON Collaboration]. arXiv:1805.12562 [astroph.CO]

77. M. Lindner, M. Platscher, F.S. Queiroz. arXiv: 1610.06587

78. F. Domingo, U. Ellwanger, JHEP 0807, 079 (2008) 